\title{
GLOBAL EXISTENCE AND UNIQUENESS OF WEAK SOLUTIONS TO CAHN-HILLIARD-GURTIN SYSTEM IN ELASTIC SOLIDS
}

\author{
IRENA PAWŁOW \\ Systems Research Institute, Polish Academy of Sciences \\ Newelska 6, 01-447 Warszawa, Poland \\ and Institute of Mathematics and Cryptology, Cybernetics Faculty \\ Military University of Technology \\ S. Kaliskiego 2, 00-908 Warszawa, Poland \\ E-mail:pawlow@ibspan.waw.pl \\ WOJCIECH M. ZAJĄCZKOWSKI \\ Institute of Mathematics, Polish Academy of Sciences \\ Sniadeckich 8, 00-956 Warszawa, Poland \\ and Institute of Mathematics and Cryptology, Cybernetics Faculty \\ Military University of Technology \\ S. Kaliskiego 2, 00-908 Warszawa, Poland \\ E-mail: wz@impan.gov.pl
}

\begin{abstract}
In this paper we study the Cahn-Hilliard-Gurtin system describing the phase-separation process in elastic solids. The system has been derived by Gurtin (1996) as an extension of the classical Cahn-Hilliard equation. For a version with viscosity we prove the existence and uniqueness of a weak solution on an infinite time interval and derive an absorbing set estimate.
\end{abstract}

1. Introduction. In this paper we study an initial-boundary-value problem for the Cahn-Hilliard system coupled with nonstationary elasticity. The system models phase separation process in deformable continuum. It was derived by Gurtin [Gur96] within the framework of his thermodynamical theory based on a microforce balance and extends the classical Cahn-Hilliard equation by elastic, anisotropic and kinetic effects.

2000 Mathematics Subject Classification: Primary 35K50; Secondary 35K60, 35Q72.

Key words and phrases: Cahn-Hilliard, nonstationary elasticity, weak solutions, global existence, uniqueness.

Research partially supported by the Polish Grant No 1 P03A 02130.

The paper is in final form and no version of it will be published elsewhere. 
Recently, various variants of this system have been often studied in the literature. In most of the studies a quasi-stationary approximation of the elasticity system, leading to a problem of elliptic-parabolic type, was used, see e.g. Garcke [Gar00], [Gar03], Gar05], Bonetti et al. [BCDGSS02], Miranville and associates, see [CarMirPR99], [CarMirP00], [Mir00], [Mir01a], [Mir01b]. The Cahn-Hilliard-Gurtin system with nonstationary elasticity leads to a problem of hyperbolic-parabolic type. It was studied in [CarMirP00], [Mir01a], [BarPaw05], [PawZaj06b] where the existence and properties of weak solutions were examined, and in [PawZaj06a], [PawZaj07] where the existence of strong solutions was proved on a finite time interval in 1-D and 3-D cases. The results of [PawZaj06a], [PawZaj06b], [PawZaj07] refer to a simplified Gurtin's model with neglected anisotropic cross-coupling terms.

In the present paper we consider the full Gurtin's model augmented in addition by mechanical and diffusive viscosity. For such problem we prove the existence and uniqueness of weak solutions on infinite time interval as well as absorbing set estimates. The obtained results allow for the long-time analysis of the problem to be presented in a separate paper.

The system under consideration consists of the following three problems for the fields of the displacement $\boldsymbol{u}: \Omega^{T} \rightarrow \mathbb{R}^{3}$, the order parameter (phase ratio) $\chi: \Omega^{T} \rightarrow \mathbb{R}$, and the chemical potential $\mu: \Omega^{T} \rightarrow \mathbb{R}:$

$$
\begin{aligned}
& \boldsymbol{u}_{t t}-\nabla \cdot\left[W_{, \varepsilon}(\varepsilon(\boldsymbol{u}), \chi)+\nu \boldsymbol{A} \varepsilon\left(\boldsymbol{u}_{t}\right)\right]=\boldsymbol{b} \quad \text { in } \Omega^{T}=\Omega \times(0, T), \\
& \left.\boldsymbol{u}\right|_{t=0}=\boldsymbol{u}_{0},\left.\quad \boldsymbol{u}_{t}\right|_{t=0}=\boldsymbol{u}_{1} \quad \text { in } \Omega \text {, } \\
& \boldsymbol{u}=\mathbf{0} \quad \text { on } S^{T}=S \times(0, T), \\
& \chi_{t}-\nabla \cdot\left(\boldsymbol{M} \nabla \mu+\boldsymbol{h}_{t}\right)=0 \quad \text { in } \Omega^{T}, \\
& \left.\chi\right|_{t=0}=\chi_{0} \quad \text { in } \Omega \text {, } \\
& \boldsymbol{n} \cdot\left(\boldsymbol{M} \nabla \mu+\boldsymbol{h} \chi_{t}\right)=0 \quad \text { on } S^{T}, \\
& \mu-\boldsymbol{g} \cdot \nabla \mu=-\nabla \cdot \boldsymbol{\Gamma} \nabla \chi+\psi^{\prime}(\chi)+W_{, \chi}(\varepsilon(\boldsymbol{u}), \chi)+\beta \chi_{t} \quad \text { in } \Omega^{T}, \\
& \boldsymbol{n} \cdot \boldsymbol{\Gamma} \nabla \chi=0 \quad \text { on } S^{T} \text {. }
\end{aligned}
$$

Here $\Omega \subset \mathbb{R}^{3}$ is a bounded domain with a smooth boundary $S$, occupied by a solid body in a reference configuration with constant mass density $\varrho=1 ; n$ is the outward unit normal to $S$ and $T>0$ is an arbitrary fixed time. The body is a binary $a-b$ alloy, which driven by thermomechanical effects, undergoes a phase separation process. Here we assume that temperature is constant below a critical value. The order parameter $\chi$ is related to the volumetric fraction of one of the two phases, characterized by different crystalline structures of the components. We assume that $\chi=-1$ is identified with the phase $a$ and $\chi=1$ with the phase $b$.

The second order tensor

$$
\varepsilon=\varepsilon(u)=\frac{1}{2}\left(\nabla \boldsymbol{u}+(\nabla \boldsymbol{u})^{T}\right)
$$

denotes the linearized strain tensor. The function $W(\varepsilon(\boldsymbol{u}), \chi)$ stands for the elastic energy, 
defined by

$$
W(\varepsilon(\boldsymbol{u}), \chi)=\frac{1}{2}(\varepsilon(\boldsymbol{u})-\bar{\varepsilon}(\chi)) \cdot \boldsymbol{A}(\varepsilon(\boldsymbol{u})-\bar{\varepsilon}(\chi)) .
$$

The corresponding derivatives

and

$$
W_{, \varepsilon}(\varepsilon(\boldsymbol{u}), \chi)=\boldsymbol{A}(\varepsilon(\boldsymbol{u})-\bar{\varepsilon}(\chi)),
$$

$$
W_{, \chi}(\varepsilon(\boldsymbol{u}), \chi)=-\bar{\varepsilon}^{\prime}(\chi) \cdot \boldsymbol{A}(\varepsilon(\boldsymbol{u})-\bar{\varepsilon}(\chi))
$$

represent respectively the stress tensor and the elastic part of the chemical potential.

The fourth order tensor $\boldsymbol{A}=\left(A_{i j k l}\right)$ denotes a constant elasticity tensor:

$$
\boldsymbol{\varepsilon}(\boldsymbol{u}) \mapsto \boldsymbol{A} \varepsilon(\boldsymbol{u})=\bar{\lambda} \operatorname{tr} \varepsilon(\boldsymbol{u}) \boldsymbol{I}+2 \bar{\mu} \varepsilon(\boldsymbol{u})
$$

where $\boldsymbol{I}=\left(\delta_{i j}\right)$ is the identity tensor, and $\bar{\lambda}, \bar{\mu}$ are the Lamé constants with values within the elasticity range (see $(2.1))$.

The second order tensor $\bar{\varepsilon}(\chi)=\left(\bar{\varepsilon}_{i j}(\chi)\right)$ denotes the eigenstrain, i.e. the stress free strain corresponding to the phase ratio $\chi$, defined by

$$
\bar{\varepsilon}(\chi)=(1-z(\chi)) \bar{\varepsilon}_{a}+z(\chi) \bar{\varepsilon}_{b},
$$

with $\bar{\varepsilon}_{a}, \bar{\varepsilon}_{b}$ denoting constant eigenstrains of phases $a, b$, and $z: \mathbb{R} \rightarrow[0,1]$ being a sufficiently smooth interpolation function satisfying

$$
z(\chi)=0 \text { for } \quad \chi \leq-1 \text { and } z(\chi)=1 \text { for } \quad \chi \geq 1 .
$$

The term $\nu \boldsymbol{A} \varepsilon\left(\boldsymbol{u}_{t}\right), \nu=$ const $\geq 0$, represents a viscous stress tensor; $\nu$ being a viscosity coefficient.

The function $\psi(\chi)$ denotes the chemical energy of the material at zero stress, assumed to be a double-well potential

$$
\psi(\chi)=\frac{1}{4}\left(1-\chi^{2}\right)^{2}
$$

with two minima at $\chi=-1$ and $\chi=1$ which characterize the phases of the material.

Further, $\boldsymbol{\Gamma}=\left(\Gamma_{i j}\right)$ is an interfacial energy tensor, and $\boldsymbol{M}=\left(M_{i j}\right)$ is a mobility tensor, both symmetric, positive definite with constant coefficients.

A constant $\beta \geq 0$ refers to a diffusional viscosity, and $\boldsymbol{g}=\left(g_{i}\right), \boldsymbol{h}=\left(h_{i}\right)$ are constant vectors accounting for anisotropic effects. In accordance with thermodynamical consistency the quantities $\boldsymbol{M}, \boldsymbol{\beta}, \boldsymbol{g}, \boldsymbol{h}$ are subject to the condition

$$
\left[\begin{array}{c}
\nabla \mu \\
\chi_{t}
\end{array}\right] \cdot\left[\begin{array}{ll}
\boldsymbol{M} & \boldsymbol{h} \\
\boldsymbol{g}^{T} & \beta
\end{array}\right]\left[\begin{array}{c}
\nabla \mu \\
\chi_{t}
\end{array}\right] \geq 0 \quad \text { for all }\left(\nabla \mu, \chi_{t}\right) \in \mathbb{R}^{3} \times \mathbb{R} .
$$

More generally, the quantities $\boldsymbol{M}, \beta, \boldsymbol{g}, \boldsymbol{h}$ may also depend on $\chi, \nabla \chi, \chi_{t}, \mu, \nabla \mu$ (see [Gur96]).

The remaining quantities in (1.1)-(1.3) have the following meaning: $\boldsymbol{b}: \Omega^{T} \rightarrow \mathbb{R}^{3}$ is an external body force, and $\boldsymbol{u}_{0}, \boldsymbol{u}_{1}: \Omega \rightarrow \mathbb{R}^{3}, \chi_{0}: \Omega \rightarrow \mathbb{R}$ are the initial conditions respectively for the displacement, the velocity and the order parameter.

The homogeneous boundary conditions in (1.1)-(1.3) are chosen for the sake of simplicity. The condition $(1.1)_{3}$ means that the body is fixed at the boundary $S,(1.2)_{3}$ reflects the mass isolation at $S$, and $(1.3)_{2}$ is the natural boundary condition for the free energy (1.10) below. 
For further use we recall a thermodynamical basis of system (1.1)-(1.3) (see e.g. [Paw06]). The underlying free energy density has the Landau-Ginzburg-Cahn-Hilliard form

$$
f(\varepsilon(\boldsymbol{u}), \chi, \nabla \chi)=W(\varepsilon(\boldsymbol{u}), \chi)+\psi(\chi)+\frac{1}{2} \nabla \chi \cdot \boldsymbol{\Gamma} \nabla \chi
$$

with the three terms on the right-hand side representing respectively the elastic, chemical and interfacial energy. Equation $(1.1)_{1}$ corresponds to the linear momentum balance

$$
\boldsymbol{u}_{t t}-\nabla \cdot \boldsymbol{S}=\boldsymbol{b}
$$

with the stress tensor $\boldsymbol{S}$ given by

$$
\boldsymbol{S}=\boldsymbol{A}(\varepsilon(\boldsymbol{u})-\bar{\varepsilon}(\chi))+\nu \boldsymbol{A} \varepsilon\left(\boldsymbol{u}_{t}\right) .
$$

Equation $(1.2)_{1}$ is the mass balance

$$
\chi_{t}+\nabla \cdot j=0
$$

with the constitutive equation for the mass flux $\boldsymbol{j}$

$$
\boldsymbol{j}=-\left(\boldsymbol{M} \nabla \mu+\boldsymbol{h} \chi_{t}\right) .
$$

Finally, equation $(1.3)_{1}$ defines a generalized chemical potential

$$
\mu=\frac{\delta f}{\delta \chi}+a,
$$

where

$$
\frac{\delta f}{\delta \chi}(\varepsilon, \chi, \nabla \chi)=f_{, \chi}(\varepsilon, \chi, \nabla \chi)-\nabla \cdot f_{, \nabla \chi}(\varepsilon, \chi, \nabla \chi)
$$

denotes the first variation of $f$ with respect to $\chi$, and $a$ is a scalar field given by

$$
a=-\left(\boldsymbol{g} \cdot \nabla \mu+\beta \chi_{t}\right) .
$$

Equivalently, in the theory of Gurtin [Gur96] equation (1.3) $)_{1}$ represents a microforce balance. In accordance with the entropy principle the quantities $\boldsymbol{j}$ and $a$ are subject to the dissipation inequality

$$
-\left(\nabla \mu \cdot \boldsymbol{j}+\chi_{t} a\right) \geq 0 \quad \text { for all }\left(\nabla \mu, \chi_{t}\right) \in \mathbb{R}^{3} \times \mathbb{R},
$$

which yields condition (1.9).

We point out that system (1.1)-(1.3) augments the original Gurtin model [Gur96] by nonstationary inertial effects $\left(\boldsymbol{u}_{t t} \neq 0\right)$ and a mechanical viscosity $(\nu>0)$.

Let us introduce now a simplified formulation of (1.1)-(1.3) obtained after taking into account the constitutive equations (1.4)-(1.6).

Let $\boldsymbol{Q}$ stand for the linear elasticity operator defined by

$$
\boldsymbol{Q u}=\nabla \cdot \boldsymbol{A} \boldsymbol{\varepsilon}(\boldsymbol{u})=\bar{\mu} \Delta \boldsymbol{u}+(\bar{\lambda}+\bar{\mu}) \nabla(\nabla \cdot \boldsymbol{u})
$$

with domain $\left.D(\boldsymbol{Q})=\boldsymbol{H}^{2} \Omega\right) \cap \boldsymbol{H}_{0}^{1}(\Omega)$. Moreover, let

$$
\Delta_{M} \mu=\nabla \cdot M \nabla \mu, \quad \Delta_{\Gamma} \chi=\nabla \cdot \Gamma \nabla \chi
$$

denote the elliptic operators associated with tensors $\boldsymbol{M}$ and $\boldsymbol{\Gamma}$. Let us define also the quantities

$$
\boldsymbol{B}=-\boldsymbol{A}\left(\overline{\boldsymbol{\varepsilon}}_{b}-\overline{\boldsymbol{\varepsilon}}_{a}\right), \quad D=-\boldsymbol{B} \cdot\left(\overline{\boldsymbol{\varepsilon}}_{b}-\overline{\boldsymbol{\varepsilon}}_{a}\right), \quad E=-\boldsymbol{B} \cdot \overline{\boldsymbol{\varepsilon}}_{a}
$$


which are respectively a symmetric second order tensor with constant coefficients and two scalars. Then

$$
\begin{aligned}
& W_{, \varepsilon}(\varepsilon(\boldsymbol{u}), \chi)=\boldsymbol{A} \varepsilon(\boldsymbol{u})-\boldsymbol{A} \bar{\varepsilon}_{a}+z(\chi) \boldsymbol{B}, \\
& W_{, \chi}(\varepsilon(\boldsymbol{u}), \chi)=z^{\prime}(\chi)(\boldsymbol{B} \cdot \boldsymbol{\varepsilon}(\boldsymbol{u})+D z(\chi)+E),
\end{aligned}
$$

and consequently (1.1)-(1.3) takes the form

$$
\begin{aligned}
& \boldsymbol{u}_{t t}-\boldsymbol{Q u}-\nu \boldsymbol{Q} \boldsymbol{u}_{t}=z^{\prime}(\chi) \boldsymbol{B} \nabla \chi+\boldsymbol{b} \quad \text { in } \Omega^{T}, \\
& \left.\boldsymbol{u}\right|_{t=0}=\boldsymbol{u}_{0},\left.\quad \boldsymbol{u}_{t}\right|_{t=0}=\boldsymbol{u}_{1} \quad \text { in } \Omega \text {, } \\
& \boldsymbol{u}=\mathbf{0} \quad \text { on } S^{T} \text {, } \\
& \chi_{t}-\nabla\left(\boldsymbol{M} \nabla \mu+\boldsymbol{h} \chi_{t}\right)=0 \quad \text { in } \Omega^{T}, \\
& \left.\chi\right|_{t=0}=\chi_{0} \quad \text { in } \Omega \text {, } \\
& \boldsymbol{n} \cdot\left(\boldsymbol{M} \nabla \mu+\boldsymbol{h} \chi_{t}\right)=0 \quad \text { on } S^{T}, \\
& \mu-\boldsymbol{g} \cdot \nabla \mu=-\nabla \cdot \boldsymbol{\Gamma} \nabla \chi+\psi^{\prime}(\chi)+z^{\prime}(\chi)(\boldsymbol{B} \cdot \boldsymbol{\varepsilon}(\boldsymbol{u})+D z(\chi)+E)+\beta \chi_{t} \quad \text { in } \Omega^{T}, \\
& \boldsymbol{n} \cdot \boldsymbol{\Gamma} \nabla \chi=0 \\
& \text { on } S^{T} \text {. }
\end{aligned}
$$

The paper is organized as follows: In Section 2 we present our main assumptions and results, stated in Theorems 2.1, 2.2, 2.3 and 2.4. Theorem 2.1 asserts the existence of a weak solution to problem $(1.1)-(1.3)$ on a fixed time interval $[0, T], T>0$. Theorems 2.2 and 2.3 provide two existence results for problem (1.1)-(1.3) on the infinite time interval. Theorem 2.2, deduced directly from a priori estimates in Theorem 2.1, requires a decay of the body force $\boldsymbol{b}$ as $t \rightarrow \infty$ and consequently implies that the dissipative quantities $\boldsymbol{u}_{t}$, $\chi_{t}$ and $\nabla \mu$ vanish in appropriate norms as $t \rightarrow \infty$. Theorem 2.3 relaxes the restrictions of Theorem 2.2 with the help of an absorbing set estimate and prolonging the solution step by step in time. Finally, Theorem 2.4 states the uniqueness of the solution to problem (1.1)-(1.3).

In Section 3 we derive basic energy estimates for (1.1)-(1.3). The procedure follows that used previously in [BarPaw05], [PawZaj06b].

In Section 4 we prove an absorbing set estimate which constitutes the main new part of the paper. This estimate allows firstly, to prolong the solution step by step on the infinite time interval and secondly, to conclude the existence of an absorbing set for system (1.1)-(1.3) which is of interest in the long-time analysis of the problem.

The subsequent sections 5 and 6 provide the proofs respectively of Theorems 2.1 and 2.2, 2.3. Finally, Section 7 gives the proof of Theorem 2.4.

We use the following notations:

$$
\begin{aligned}
& \boldsymbol{x}=\left(x_{i}\right)_{i=1,2,3} \text { the material point, } \\
& f_{, i}=\frac{\partial f}{\partial x_{i}}, \quad f_{t}=\frac{\partial f}{\partial t} \text { the material space and time derivatives, } \\
& \boldsymbol{\varepsilon}=\left(\varepsilon_{i j}\right)_{i, j=1,2,3}, \quad W_{, \varepsilon}(\varepsilon, \chi)=\left(\frac{\partial W(\varepsilon, \chi)}{\partial \varepsilon_{i j}}\right)_{i, j=1,2,3}, \\
& W_{, \chi}(\varepsilon, \chi)=\frac{\partial W(\varepsilon, \chi)}{\partial \chi}, \quad \psi^{\prime}(\chi)=\frac{d \psi(\chi)}{d \chi}
\end{aligned}
$$


For simplicity, whenever there is no danger of confusion, we omit the arguments $(\varepsilon, \chi)$. The specification of tensor indices in omitted as well. Vector- and tensor-valued mappings are denoted by bold letters. The summation convention over repeated indices is used, as well as the notation: for vectors $\boldsymbol{a}=\left(a_{i}\right), \tilde{\boldsymbol{a}}=\left(a_{i}\right)$ and tensors $\boldsymbol{B}=\left(B_{i j}\right), \tilde{\boldsymbol{B}}=\left(\tilde{B}_{i j}\right)$, $\boldsymbol{A}=\left(A_{i j k l}\right)$, we write

$$
\begin{array}{ll}
\boldsymbol{a} \cdot \tilde{\boldsymbol{a}}=a_{i} \tilde{a}_{i}, & \boldsymbol{B} \cdot \tilde{\boldsymbol{B}}=B_{i j} \tilde{B}_{i j}, \\
\boldsymbol{A} \boldsymbol{B}=\left(A_{i j k l} B_{k l}\right), & \boldsymbol{B} \boldsymbol{A}=\left(B_{i j} A_{i j k l}\right), \\
|\boldsymbol{a}|=\left(a_{i} a_{i}\right)^{1 / 2}, & |\boldsymbol{B}|=\left(B_{i j} B_{i j}\right)^{1 / 2} .
\end{array}
$$

The symbols $\nabla$ and $\nabla \cdot$ denote the gradient and the divergence operators with respect to the material point $\boldsymbol{x}$. For the divergence of a tensor field we use the convention of the contraction over the last index, e.g. $\nabla \cdot \varepsilon(\boldsymbol{x})=\left(\varepsilon_{i j, j}(\boldsymbol{x})\right)$.

We use the standard Sobolev spaces notation $H^{m}(\Omega)=W_{2}^{m}(\Omega)$ for $m \in \mathbb{N}$. Moreover,

$$
\begin{aligned}
& H_{0}^{1}(\Omega)=\left\{v \in H^{1}(\Omega): v=0 \text { on } S\right\}, \\
& H_{N}^{2}(\Omega)=\left\{v \in H^{2}(\Omega): \boldsymbol{n} \cdot \boldsymbol{\Gamma} \nabla v=0 \text { on } S\right\},
\end{aligned}
$$

where $\boldsymbol{n}$ is the outward unit normal to $S=\partial \Omega$, denote the subspaces respectively of $H^{1}(\Omega)$ and $H^{2}(\Omega)$, with the standard norms of $H^{1}(\Omega)$ and $H^{2}(\Omega)$.

By bold letters we denote the spaces of vector- or tensor-valued functions, e.g.

$$
\boldsymbol{L}_{2}(\Omega)=\left(L_{2}(\Omega)\right)^{n}, \quad \boldsymbol{H}^{1}(\Omega)=\left(H^{1}(\Omega)\right)^{n}, \quad n \in \mathbb{N} ;
$$

if there is no confusion we do not specify dimension $n$. Moreover, we write

$$
\|\boldsymbol{a}\|_{L_{2}(\Omega)}=\||\boldsymbol{a}|\|_{L_{2}(\Omega)}, \quad\|\boldsymbol{a}\|_{\boldsymbol{H}^{1}(\Omega)}=\||\boldsymbol{a}|\|_{L_{2}(\Omega)}+\||\nabla \boldsymbol{a}|\|_{L_{2}(\Omega)}
$$

for the corresponding norms of a vector-valued function $\boldsymbol{a}(\boldsymbol{x})=\left(a_{i}(\boldsymbol{x})\right)$; similarly for tensor-valued functions.

As usual, the symbol $(\cdot, \cdot)$ denotes the scalar product in $L_{2}(\Omega)$. For simplicity, we use the same symbol to denote scalar products in $\boldsymbol{L}_{2}(\Omega)=\left(L_{2}(\Omega)\right)^{n}$, e.g. we write

$$
(a, \tilde{a})=\int_{\Omega} a(\boldsymbol{x}) \tilde{a}(\boldsymbol{x}) d x, \quad(\boldsymbol{a}, \tilde{\boldsymbol{a}})=\int_{\Omega} a_{i}(\boldsymbol{x}) \tilde{a}_{i}(\boldsymbol{x}) d x, \quad(\boldsymbol{B}, \tilde{\boldsymbol{B}})=\int_{\Omega} B_{i j}(\boldsymbol{x}) \tilde{B}_{i j}(\boldsymbol{x}) d x .
$$

The dual of the space $V$ is denoted by $V^{\prime}$, and $\langle\cdot, \cdot\rangle_{V^{\prime}, V}$ stands for the duality pairing between $V^{\prime}$ and $V$.

By $c$ and $c(T)$ we denote generic positive constants different in various instances, depending on the data of the problem and domain $\Omega$; whenever it is of interest their dependence on parameters is specified. The argument $T$ indicates the time horizon dependence. Moreover, $\delta$ denotes a generic, sufficiently small positive constant.

For further use we collect also some frequently used inequalities. The first one is the Korn inequality

$$
\|\boldsymbol{u}\|_{\boldsymbol{H}^{1}(\Omega)} \leq d_{1}^{-1 / 2}\|\boldsymbol{\varepsilon}(\boldsymbol{u})\|_{\boldsymbol{L}_{2}(\Omega)} \quad \text { for } \quad \boldsymbol{u} \in \boldsymbol{H}_{0}^{1}(\Omega)
$$

with a positive constant $d_{1}$. The second one is the Poincaré inequality

$$
\int_{\Omega}\left|\chi-f_{\Omega} \chi d x^{\prime}\right|^{2} d x \leq d_{2}\|\nabla \chi\|_{\boldsymbol{L}_{2}(\Omega)}^{2} \quad \text { for } \quad \chi \in H^{1}(\Omega)
$$


where $d_{2}$ is a positive constant, and $f_{\Omega} \chi d x$ denotes the mean value of $\chi$ :

$$
f_{\Omega} \chi d x=\frac{1}{|\Omega|} \int_{\Omega} \chi d x, \quad|\Omega|=\text { meas } \Omega .
$$

The third one is the Poincaré-Friedrichs inequality

$$
\|\boldsymbol{u}\|_{\boldsymbol{L}_{2}(\Omega)} \leq d_{3}^{1 / 2}\|\nabla \boldsymbol{u}\|_{\boldsymbol{L}_{2}(\Omega)} \quad \text { for } \boldsymbol{u} \in \boldsymbol{H}_{0}^{1}(\Omega)
$$

with a positive constant $d_{3}$. For completeness we recall also the Sobolev imbedding

$$
\|\chi\|_{L_{6}(\Omega)} \leq d_{4}^{1 / 2}\|\chi\|_{H^{1}(\Omega)}
$$

with a positive constant $d_{4}$.

2. Assumptions and main results. System (1.1)-(1.3) (in simplified form (1.15)(1.17)) is studied under the following assumptions:

(A1) $\Omega \subset \mathbb{R}^{3}$ is a bounded domain with the boundary $S$ of class at least $C^{2} ; T>0$ is an arbitrary fixed time.

(A2) The coefficients of the elasticity operator $\boldsymbol{Q}$ (see (1.11)) satisfy

$$
\bar{\mu}>0, \quad 3 \bar{\lambda}+2 \bar{\mu}>0 \text { (elasticity range). }
$$

These two conditions assure the following:

(i) the elasticity tensor $\boldsymbol{A}$ is coercive and bounded

$$
c_{*}|\varepsilon|^{2} \leq \varepsilon \cdot \boldsymbol{A} \varepsilon \leq c^{*}|\varepsilon|^{2} \text { for all } \varepsilon \in \boldsymbol{S}^{2},
$$

where $\boldsymbol{S}^{2}$ denotes the set of symmetric second order tensors in $\mathbb{R}^{3}$, and

$$
c_{*}=\min \{3 \bar{\lambda}+2 \bar{\mu}, 2 \bar{\mu}\}, \quad c^{*}=\max \{3 \bar{\lambda}+2 \bar{\mu}, 2 \bar{\mu}\} ;
$$

(ii) The operator $Q$ is strongly elliptic and satisfies the estimate

$$
c\|\boldsymbol{u}\|_{\boldsymbol{H}^{2}(\Omega)} \leq\|\boldsymbol{Q} \boldsymbol{u}\|_{\boldsymbol{L}_{2}(\Omega)} \quad \text { for } \quad \boldsymbol{u} \in D(\boldsymbol{Q})=\boldsymbol{H}^{2}(\Omega) \cap \boldsymbol{H}_{0}^{1}(\Omega)
$$

with constant $c$ depending on $\Omega$;

(A3) The mechanical viscosity coefficient is positive $\nu=$ const $>0$.

The next three assumptions concern the ingredients of the free energy $f(\varepsilon(\boldsymbol{u}), \chi, \nabla \chi)$ in $(1.10)$.

(A4) The elastic energy $W(\varepsilon(\boldsymbol{u}), \chi)$ is fiven by (1.4)-(1.6) with the interpolation function $z: \mathbb{R} \rightarrow[0,1]$ being at least of class $C^{1}$, satisfying (1.7) and such that

$$
\left|z^{\prime}(\chi)\right| \leq c \text { for all } \chi \in \mathbb{R} .
$$

The auxiliary constant quantities $\boldsymbol{B}, D$ and $E$ are defined in (1.13).

(A5) The chemical energy is a double-well potential (1.8), so that

$$
\psi^{\prime}(\chi)=\chi^{3}-\chi, \quad \psi^{\prime \prime}(\chi)=3 \chi^{2}-1, \quad \psi^{\prime \prime \prime}(\chi)=6 \chi .
$$

(A6) The interfacial energy tensor $\boldsymbol{\Gamma}=\left(\Gamma_{i j}\right)$ is symmetric, with constant coefficients, positive definite and bounded:

$$
\underline{c}_{\Gamma}|\boldsymbol{\xi}|^{2} \leq \boldsymbol{\xi} \cdot \boldsymbol{\Gamma} \boldsymbol{\xi} \leq \bar{c}_{\Gamma}|\boldsymbol{\xi}|^{2} \forall \boldsymbol{\xi} \in \mathbb{R}^{3}
$$


with constants $0<\underline{c}_{\Gamma}<\bar{c}_{\Gamma}$. This implies that the operator $\Delta_{\Gamma}$ defined in (1.12) is strongly elliptic.

We note that, in view of (1.14), it follows from (A4) that there exist positive constants $a_{1}, a_{2}$ such that

$$
\left|W_{, \chi}(\varepsilon, \chi)\right| \leq a_{1}(|\varepsilon|+1), \quad\left|W_{, \varepsilon}(\varepsilon, \chi)\right| \leq a_{2}(|\varepsilon|+1)
$$

for all $\varepsilon \in S^{2}$ and $\chi \in \mathbb{R}$. Moreover, on account of (2.2) and (1.8), the Young inequality implies that

$$
W(\varepsilon(\boldsymbol{u}), \chi) \geq \frac{c_{*}}{2}|\varepsilon(\boldsymbol{u})-\bar{\varepsilon}(\chi)|^{2} \geq \frac{c_{*}}{2}\left(\frac{1}{2}|\varepsilon(\boldsymbol{u})|^{2}-|\bar{\varepsilon}(\chi)|^{2}\right)
$$

and

$$
\psi(\chi) \geq \frac{1}{8} \chi^{4}-\frac{1}{4}
$$

We note also that thanks to assumption (A4) there exist positive constants $a_{3}, a_{4}$ such that

$$
|\bar{\varepsilon}(\chi)| \leq a_{3}, \quad\left|\bar{\varepsilon}^{\prime}(\chi)\right|+\left|\bar{\varepsilon}^{\prime}(\chi) \chi\right| \leq a_{4} .
$$

The remaining assumptions refer to the quantities $\boldsymbol{M}, \beta, \boldsymbol{g}$ and $\boldsymbol{h}$.

(A7) The mobility tensor $\boldsymbol{M}=\left(M_{i j}\right)$ is symmetric, with constant coefficients, positive definite and bounded:

$$
\underline{c}_{M}|\boldsymbol{\xi}|^{2} \leq \boldsymbol{\xi} \cdot \boldsymbol{M} \boldsymbol{\xi} \leq \bar{c}_{M}|\boldsymbol{\xi}|^{2} \quad \forall \boldsymbol{\xi} \in \mathbb{R}^{3}
$$

with constants $0<\underline{c}_{M}<\bar{c}_{M}$. This implies that the operator $\Delta_{M}$ is strongly elliptic.

(A8) The diffusional viscosity coefficient is positive $\beta=$ const $>0$.

(A9) The vectors $\boldsymbol{g}$ and $\boldsymbol{h}$ are constant and such that the matrix

$$
\boldsymbol{P}:=\left[\begin{array}{cc}
\boldsymbol{M} & \boldsymbol{h} \\
\boldsymbol{g}^{T} & \beta
\end{array}\right]
$$

is strictly positive definite in the sense that there exists a constant $c_{P}>0$ such that

$$
\begin{aligned}
\boldsymbol{X} \cdot \boldsymbol{P} \boldsymbol{X}=\nabla \mu \cdot \boldsymbol{M} \nabla \mu+\chi_{t}(\boldsymbol{g}+\boldsymbol{h}) \cdot \nabla \mu+\beta \chi_{t}^{2} & \\
& \geq c_{P}\left(|\nabla \mu|^{2}+\left|\chi_{t}\right|^{2}\right) \quad \forall \boldsymbol{X}=\left(\nabla \mu, \chi_{t}\right) \in \mathbb{R}^{3} \times \mathbb{R} .
\end{aligned}
$$

We state now the results of the paper. The first theorem asserts the existence of a weak solution to problem (1.1)-(1.3) on the interval $[0, T], T>0$. It modifies the result obtained in [BarPaw05], Thm 3.2.

Theorem 2.1 (Existence on $[0, T], T>0)$. Let the assumptions (A1)-(A9) hold. Moreover, let the data satisfy

$$
\boldsymbol{b} \in L_{2}\left(0, T ; \boldsymbol{L}_{2}(\Omega)\right), \quad \boldsymbol{u}_{0} \in \boldsymbol{H}_{0}^{1}(\Omega), \quad \boldsymbol{u}_{1} \in \boldsymbol{L}_{2}(\Omega), \quad \chi_{0} \in H^{1}(\Omega) .
$$


Then there exist functions $(\boldsymbol{u}, \chi, \mu)$ such that:

$$
\begin{aligned}
& \boldsymbol{u} \in L_{\infty}\left(0, T ; \boldsymbol{H}_{0}^{1}(\Omega)\right), \quad \boldsymbol{u}_{t} \in L_{\infty}\left(0, T ; \boldsymbol{L}_{2}(\Omega) \cap L_{2}\left(0, T ; \boldsymbol{H}_{0}^{1}(\Omega)\right),\right. \\
& \boldsymbol{u}_{t t} \in L_{2}\left(0, T ;\left(\boldsymbol{H}_{0}^{1}(\Omega)\right)^{\prime}\right), \\
& \chi \in L_{\infty}\left(0, T ; H^{1}(\Omega)\right) \cap L_{2}\left(0, T ; H_{N}^{2}(\Omega)\right), \quad \chi_{t} \in L_{2}\left(\Omega^{T}\right), \\
& \int_{\Omega} \chi(t) d x=\int_{\Omega} \chi_{0} d x \equiv \chi_{m} \quad \text { for } t \in[0, T], \quad \mu \in L_{2}\left(0, T ; H^{1}(\Omega)\right),
\end{aligned}
$$

where $H_{N}^{2}(\Omega)=\left\{\chi \in H^{2}(\Omega): \boldsymbol{n} \cdot \boldsymbol{\Gamma} \nabla \chi=0\right.$ on $\left.S\right\}$,

$$
\boldsymbol{u}(0)=\boldsymbol{u}_{0}, \quad \boldsymbol{u}_{t}(0)=\boldsymbol{u}_{1}, \quad \chi(0)=\chi_{0},
$$

which satisfy problem (1.15)-(1.17) in the following weak sense

$$
\begin{aligned}
& \int_{0}^{T}\left\langle\boldsymbol{u}_{t t}, \boldsymbol{\eta}\right\rangle_{\left(\boldsymbol{H}_{0}^{1}(\Omega)\right)^{\prime}, \boldsymbol{H}_{0}^{1}(\Omega)} d t+\int_{0}^{T}(\boldsymbol{A} \boldsymbol{\varepsilon}(\boldsymbol{u}), \boldsymbol{\varepsilon}(\boldsymbol{\eta})) d t \\
& \quad+\nu \int_{0}^{T}\left(\boldsymbol{A} \boldsymbol{\varepsilon}\left(\boldsymbol{u}_{t}\right), \boldsymbol{\varepsilon}(\boldsymbol{\eta})\right) d t=\int_{0}^{T}\left(z^{\prime}(\chi) \boldsymbol{B} \nabla \chi+\boldsymbol{b}, \boldsymbol{\eta}\right) d t \\
& \quad \forall \boldsymbol{\eta} \in L_{2}\left(0, T ; \boldsymbol{H}_{0}^{1}(\Omega)\right),
\end{aligned}
$$$$
\int_{0}^{T}\left(\chi_{t}, \xi\right) d t+\int_{0}^{T}\left(\boldsymbol{M} \nabla \mu+\boldsymbol{h} \chi_{t}, \nabla \xi\right) d t=0
$$$$
\forall \xi \in C^{1}\left([0, T] ; H^{1}(\Omega)\right) \quad \xi(T)=0,
$$$$
\int_{0}^{T}(\mu-\boldsymbol{g} \cdot \nabla \mu, \zeta) d t=-\int_{0}^{T}\left(\Delta_{\boldsymbol{\Gamma}} \chi, \zeta\right) d t
$$$$
+\int_{0}^{T}\left(\psi^{\prime}(\chi)+z^{\prime}(\chi)(\boldsymbol{B} \cdot \varepsilon(\boldsymbol{u})+D z(\chi)+E), \zeta\right) d t \quad \forall \zeta \in L_{2}\left(0, T ; L_{2}(\Omega)\right) .
$$

Moreover, $(\boldsymbol{u}, \chi, \mu)$ satisfy a priori estimates:

- estimates uniform in T:

$$
\begin{aligned}
\frac{1}{2}\left\|\boldsymbol{u}_{t}\right\|_{L_{\infty}\left(0, T ; \boldsymbol{L}_{2}(\Omega)\right)}^{2}+c_{1}\|\boldsymbol{u}\|_{L_{\infty}\left(0, T ; \boldsymbol{H}_{0}^{1}(\Omega)\right)}^{2} & \\
& +\frac{1}{2} \underline{c}_{\Gamma}\|\nabla \chi\|_{L_{\infty}\left(0, T ; \boldsymbol{L}_{2}(\Omega)\right)}^{2}+\frac{1}{8}\|\chi\|_{L_{\infty}\left(0, T ; L_{4}(\Omega)\right)}^{4} \\
& +c_{P}\|\nabla \mu\|_{\boldsymbol{L}_{2}\left(\Omega^{T}\right)}^{2}+c_{P}\left\|\chi_{t}\right\|_{L_{2}\left(\Omega^{T}\right)}^{2}+\nu c_{*}\left\|\varepsilon\left(\boldsymbol{u}_{t}\right)\right\|_{\boldsymbol{L}_{2}\left(\Omega^{T}\right)}^{2} \leq \alpha_{1}
\end{aligned}
$$

where

$$
\begin{gathered}
\alpha_{1}=2 F(0)+\frac{3}{2}\|\boldsymbol{b}\|_{L_{1}\left(0, T ; L_{2}(\Omega)\right)}^{2}+c_{2} \\
F(0)=\int_{\Omega}\left[\frac{1}{2}\left(\left|\boldsymbol{u}_{1}\right|^{2}+\nabla \chi_{0} \cdot \boldsymbol{\Gamma} \nabla \chi_{0}\right)+\psi\left(\chi_{0}\right)+W\left(\varepsilon\left(\boldsymbol{u}_{0}\right), \chi_{0}\right)\right] d x \\
c_{1}=c_{*} d_{1} / 4, \quad c_{2}=\left(2 c_{*} a_{3}^{2}+1\right)|\Omega| / 4
\end{gathered}
$$

with constants $d_{1}, a_{3}$ defined in (1.18), (2.10), $c_{*}$ in (2.2); 
- estimates depending on $T$

$$
\begin{aligned}
& \|\mu\|_{L_{2}\left(0, T ; H^{1}(\Omega)\right)}^{2} \leq c_{4}(1+T), \\
& \|\chi\|_{L_{2}\left(0, T ; H^{2}(\Omega)\right)}^{2} \leq c_{5}(1+T), \\
& \left\|\boldsymbol{u}_{t t}\right\|_{L_{2}\left(0, T ;\left(\boldsymbol{H}_{0}^{1}(\Omega)\right)^{\prime}\right)}^{2} \leq c_{6}(1+T),
\end{aligned}
$$

where constant $c_{4}$ depends on $\alpha_{1}$, constant $c_{5}$ on $\alpha_{1}$ and $\chi_{m}$, and constant $c_{6}$ on $\alpha_{1}$ and $\|\boldsymbol{b}\|_{L_{2}\left(0, T ; \boldsymbol{L}_{2}(\Omega)\right)}$.

The next two theorems provide global existence results for problem (1.1)-(1.3). The first one follows directly from the estimates for weak solutions in Theorem 2.1. Such approach implies that $\boldsymbol{b}, \boldsymbol{u}_{t}, \chi_{t}$ and $\nabla \mu$ vanish in appropriate norms as $t \rightarrow \infty$. The second theorem relaxes these restrictions. This is possible by additional absorbing set estimate and prolonging the solution step by step in time.

Theorem 2.2 (The first global existence). Assume the hypotheses of Theorem 2.1 hold and

$$
\begin{aligned}
& \boldsymbol{b} \in L_{1}\left(\mathbb{R}_{+} ; \boldsymbol{L}_{2}(\Omega)\right), \\
& \sup _{k \in \mathbb{N} \cup\{0\}}\|\boldsymbol{b}\|_{L_{2}\left(k T,(k+1) T ; \boldsymbol{L}_{2}(\Omega)\right)}<\infty, \\
& \boldsymbol{u}_{0} \in \boldsymbol{H}^{1}(\Omega), \boldsymbol{u}_{1} \in \boldsymbol{L}_{2}(\Omega), \chi_{0} \in H^{1}(\Omega) .
\end{aligned}
$$

Then there exists a global solution to (1.1)-(1.3) such that

$$
\begin{aligned}
& \boldsymbol{u} \in L_{\infty}\left(\mathbb{R}_{+} ; \boldsymbol{H}_{0}^{1}(\Omega)\right), \boldsymbol{u}_{t} \in L_{\infty}\left(\mathbb{R}_{+} ; \boldsymbol{L}_{2}(\Omega)\right) \cap L_{2}\left(\mathbb{R}_{+} ; \boldsymbol{H}_{0}^{1}(\Omega)\right), \\
& \chi \in L_{\infty}\left(\mathbb{R}_{+} ; H^{1}(\Omega)\right), \chi_{t} \in L_{2}\left(\mathbb{R}_{+} ; L_{2}(\Omega)\right), \quad \int_{\Omega} \chi(t) d x=\int_{\Omega} \chi_{0} d x, \\
& \nabla \mu \in L_{2}\left(\mathbb{R}_{+} ; \boldsymbol{L}_{2}(\Omega)\right),
\end{aligned}
$$

satisfying the following estimates:

- estimates uniform in time:

$$
\begin{aligned}
& \frac{1}{2}\left\|\boldsymbol{u}_{t}\right\|_{L_{\infty}\left(\mathbb{R}_{+} ; \boldsymbol{L}_{2}(\Omega)\right)}^{2}+c_{1}\|\boldsymbol{u}\|_{L_{\infty}\left(\mathbb{R}_{+} ; \boldsymbol{H}_{0}^{1}(\Omega)\right)}^{2}+\frac{1}{2} \underline{c}_{\Gamma}\|\nabla \chi\|_{L_{\infty}\left(\mathbb{R}_{+} ; \boldsymbol{L}_{2}(\Omega)\right)}^{2} \\
& \quad+\frac{1}{8}\|\chi\|_{L_{\infty}\left(\mathbb{R}_{+} ; L_{4}(\Omega)\right)}^{4}+c_{P}\|\nabla \mu\|_{L_{2}\left(\mathbb{R}_{+} ; \boldsymbol{L}_{2}(\Omega)\right)}^{2} \\
& \quad+c_{P}\left\|\chi_{t}\right\|_{L_{2}\left(\mathbb{R}_{+} ; L_{2}(\Omega)\right)}^{2}+\nu c_{*}\left\|\varepsilon\left(\boldsymbol{u}_{t}\right)\right\|_{L_{2}\left(\mathbb{R}_{+} ; \boldsymbol{L}_{2}(\Omega)\right)} \leq \tilde{\alpha}_{1}
\end{aligned}
$$

where

$$
\tilde{\alpha}_{1}=2 F(0)+\frac{3}{2}\|\boldsymbol{b}\|_{L_{1}\left(\mathbb{R}_{+} ; \boldsymbol{L}_{2}(\Omega)\right)}^{2}+c_{2} ;
$$

- for any $t \in \mathbb{R}_{+}$and any fixed $T>0$

$$
\begin{aligned}
& \|\mu\|_{L_{2}\left(t, t+T ; H^{1}(\Omega)\right)}^{2} \leq c_{4}(1+T), \\
& \|\chi\|_{L_{2}\left(t, t+T ; H^{2}(\Omega)\right)}^{2} \leq c_{5}(1+T), \\
& \left\|\boldsymbol{u}_{t t}\right\|_{L_{2}\left(t, t+T ;\left(\boldsymbol{H}_{0}^{1}(\Omega)\right)^{\prime}\right)}^{2} \leq \tilde{c}_{6}(1+T)
\end{aligned}
$$

with constants $c_{4}, c_{5}$ as in Theorem 2.1, and constant $\tilde{c}_{6}$ depending on $\alpha_{1}$ and

$$
\sup _{k \in \mathbb{N} \cup\{0\}}\|\boldsymbol{b}\|_{L_{2}\left(k T,(k+1) T ; \boldsymbol{L}_{2}(\Omega)\right)} .
$$


THEOREM 2.3 (The second global existence). Assume the hypotheses of Theorem 2.1 and

$$
\sup _{k \in \mathbb{N} \cup\{0\}}\|\boldsymbol{b}\|_{L_{2}\left(k T ;(k+1) T ; \boldsymbol{L}_{2}(\Omega)\right)}<\infty, \quad \boldsymbol{u}_{0} \in \boldsymbol{H}^{1}(\Omega), \boldsymbol{u}_{1} \in \boldsymbol{L}_{2}(\Omega), \chi_{0} \in H^{1}(\Omega) .
$$

Then there exists a global solution to problem (1.1)-(1.3) such that

$$
\begin{gathered}
A_{1} \equiv\|\boldsymbol{u}\|_{L_{\infty}\left(\mathbb{R}_{+} ; \boldsymbol{H}_{0}^{1}(\Omega)\right)}+\left\|\boldsymbol{u}_{t}\right\|_{L_{\infty}\left(\mathbb{R}_{+} ; \boldsymbol{L}_{2}(\Omega)\right)}+\|\chi\|_{L_{\infty}\left(\mathbb{R}_{+} ; H^{1}(\Omega)\right)}<\infty \\
A_{2} \equiv \sup _{k \in \mathbb{N} \cup\{0\}}\left(\left\|\boldsymbol{u}_{t}\right\|_{L_{2}\left(k T,(k+1) T ; \boldsymbol{H}_{0}^{1}(\Omega)\right)}+\left\|\chi_{t}\right\|_{L_{2}\left(k T ;(k+1) T ; L_{2}(\Omega)\right)}\right. \\
\left.+\|\nabla \mu\|_{L_{2}\left(k T,(k+1) T ; \boldsymbol{L}_{2}(\Omega)\right)}\right)<\infty \\
\int_{\Omega} \chi(t) d x=\int_{\Omega} \chi_{0} d x,
\end{gathered}
$$

where

$$
A_{1}+A_{2} \leq 2 F(0)+\frac{3}{2} T \sup _{k \in \mathbb{N} \cup\{0\}}\|\boldsymbol{b}\|_{L_{2}\left(k T,(k+1) T ; \boldsymbol{L}_{2}(\Omega)\right)}^{2}+c_{2}
$$

Moreover,

$$
\begin{aligned}
& \sup _{k \in \mathbb{N} \cup\{0\}}\|\mu\|_{L_{2}\left(k T,(k+1) T ; H^{1}(\Omega)\right)} \leq c_{4}(1+T), \\
& \sup _{k \in \mathbb{N} \cup\{0\}}\|\chi\|_{L_{2}\left(k T,(k+1) T ; H^{2}(\Omega)\right)} \leq c_{5}(1+T), \\
& \sup _{k \in \mathbb{N} \cup\{0\}}\left\|\boldsymbol{u}_{t t}\right\|_{L_{2}\left(k T,(k+1) T ;\left(\boldsymbol{H}_{0}^{1}(\Omega)\right)^{\prime}\right)} \leq \tilde{c}_{6}(1+T)
\end{aligned}
$$

with constants $c_{4}, c_{5}, \tilde{c}_{6}$ as in Theorem 2.2 .

Theorem 2.4 (The uniqueness). Assume the hypotheses of Theorem 2.1 and $z \in C^{2}(\mathbb{R})$. Then the solution to problem (1.1)-(1.3) is unique.

3. Energy estimates. In this section we derive basic energy estimates for problem (1.1)-(1.3). These estimates imply the existence of solutions on a fixed time interval $[0, T]$, asserted in Theorem 2.1. For the clarity of presentation we shall derive only formal estimates. The presented estimates can be made rigorous by considering a Faedo-Galerkin approximation and by passing to the limit with approximation by standard compactness arguments in a similar fashion as e.g. in [PawZaj06b], [PawZaj07].

To see the influence of the data on energy estimates and later in Section 4 on absorbing set estimates we record explicitly the data-dependences of all appearing constants.

Firstly we shall show the energy identity for system (1.1)-(1.3). Let

$$
\begin{aligned}
F(t) & =\int_{\Omega}\left[\frac{1}{2}\left|\boldsymbol{u}_{t}\right|^{2}+f(\varepsilon(\boldsymbol{u}), \chi, \nabla \chi)\right] d x \\
& =\int_{\Omega}\left[\frac{1}{2}\left(\left|\boldsymbol{u}_{t}\right|^{2}+\nabla \chi \cdot \Gamma \nabla \chi\right)+\psi(\chi)+W(\varepsilon(\boldsymbol{u}), \chi)\right] d x
\end{aligned}
$$

denote the total energy of (1.1)-(1.3). We have

LEMMA 3.1. Let $(\boldsymbol{u}, \chi, \mu)$ be a sufficiently regular solution to problem (1.1)-(1.3), and $F$ be given by (3.1). Then the following equality is valid 


$$
\begin{aligned}
\frac{d F}{d t} & +\nu \int_{\Omega} \boldsymbol{\varepsilon}\left(\boldsymbol{u}_{t}\right) \cdot \boldsymbol{A} \varepsilon\left(\boldsymbol{u}_{t}\right) d x \\
& +\int_{\Omega}\left[\nabla \mu \cdot \boldsymbol{M} \nabla \mu+\chi_{t}(\boldsymbol{g}+\boldsymbol{h}) \cdot \nabla \mu+\beta \chi_{t}^{2}\right] d x=\int_{\Omega} \boldsymbol{b} \cdot \boldsymbol{u}_{t} d x \quad \text { for } t \in[0, T] .
\end{aligned}
$$

Proof. Multiplying $(1.1)_{1}$ by $\boldsymbol{u}_{t}$, integrating over $\Omega$ and by parts, using boundary condition $(1.1)_{3}$, it follows that

$$
\begin{gathered}
\frac{1}{2} \frac{d}{d t} \int_{\Omega}\left|\boldsymbol{u}_{t}\right|^{2} d x+\int_{\Omega} W_{, \varepsilon}(\varepsilon(\boldsymbol{u}), \chi) \cdot \boldsymbol{\varepsilon}\left(\boldsymbol{u}_{t}\right) d x \\
+\nu \int_{\Omega} \boldsymbol{\varepsilon}\left(\boldsymbol{u}_{t}\right) \cdot \boldsymbol{A} \boldsymbol{\varepsilon}\left(\boldsymbol{u}_{t}\right) d x=\int_{\Omega} \boldsymbol{b} \cdot \boldsymbol{u}_{t} d x .
\end{gathered}
$$

Further, testing $(1.2)_{1}$ with $\mu$, integrating over $\Omega$ and by parts, using $(1.2)_{3}$, yields

$$
\int_{\Omega} \chi_{t} \mu d x+\int_{\Omega}\left(\boldsymbol{M} \nabla \mu+\boldsymbol{h} \chi_{t}\right) \cdot \nabla \mu d x=0 .
$$

Finally, testing $(1.3)_{1}$ with $-\chi_{t}$, integrating by parts and using $(1.3)_{2}$, leads to

$$
\begin{aligned}
& -\int_{\Omega} \mu \chi_{t} d x+\int_{\Omega} \chi_{t} \boldsymbol{g} \cdot \nabla \mu d x+\frac{1}{2} \frac{d}{d t} \int_{\Omega} \nabla \chi \cdot \Gamma \nabla \chi d x+\int_{\Omega} \psi^{\prime}(\chi) \chi_{t} d x \\
& \quad+\int_{\Omega} W_{, \chi}(\varepsilon(\boldsymbol{u}), \chi) \chi_{t} d x+\beta \int_{\Omega} \chi_{t}^{2} d x=0 .
\end{aligned}
$$

Summing up (3.3)-(3.5) gives (3.2) and thereby completes the proof.

From Lemma 3.1 we deduce the following basic energy estimate.

Lemma 3.2. Assume (A1)-(A9) hold, $F$ is given by (3.1), and $\boldsymbol{b} \in L_{1}\left(0, T ; \boldsymbol{L}_{2}(\Omega)\right)$. Then

$$
\begin{aligned}
& \frac{1}{2}\left(\left\|\boldsymbol{u}_{t}\right\|_{\boldsymbol{L}_{2}(\Omega)}^{2}+\underline{c}_{\Gamma}\|\nabla \chi\|_{\boldsymbol{L}_{2}(\Omega)}^{2}\right)+c_{1}\|\boldsymbol{u}\|_{\boldsymbol{H}^{1}(\Omega)}^{2}+\frac{1}{8}\|\chi\|_{L_{4}(\Omega)}^{4} \\
& \quad+c_{P} \int_{0}^{t}\left(\|\nabla \mu\|_{\boldsymbol{L}_{2}(\Omega)}^{2}+\left\|\chi_{t^{\prime}}\right\|_{L_{2}(\Omega)}^{2}\right) d t^{\prime}+\nu c_{*} \int_{0}^{t}\left\|\boldsymbol{\varepsilon}\left(\boldsymbol{u}_{t^{\prime}}\right)\right\|_{\boldsymbol{L}_{2}(\Omega)}^{2} d t^{\prime} \\
& \leq F(t)+c_{P} \int_{0}^{t}\left(\|\nabla \mu\|_{\boldsymbol{L}_{2}(\Omega)}^{2}+\left\|\chi_{t^{\prime}}\right\|_{L_{2}(\Omega)}^{2}\right) d t^{\prime}+\nu c_{*} \int_{0}^{t}\left\|\boldsymbol{\varepsilon}\left(\boldsymbol{u}_{t^{\prime}}\right)\right\|_{\boldsymbol{L}_{2}(\Omega)}^{2} d t^{\prime} \\
& \leq 2 F(0)+\frac{3}{2}\|\boldsymbol{b}\|_{L_{1}\left(0, T ; \boldsymbol{L}_{2}(\Omega)\right)}^{2}+c_{2} \equiv \alpha_{1} \quad \text { for } t \in[0, T],
\end{aligned}
$$

with positive constants $c_{1}, c_{2}$ independent of $T$, given by

$$
c_{1}=\frac{c_{*}}{4} d_{1}, \quad c_{2}=\frac{1}{2}\left(c_{*} a_{3}^{2}+\frac{1}{2}\right)|\Omega| .
$$

Proof. We apply the Hölder inequality to the right-hand side of (3.2), use the definition of $F$, and conditions $(2.2),(2.12)$ to conclude

$$
\begin{aligned}
& \frac{d}{d t} F+\nu c_{*}\left\|\varepsilon\left(\boldsymbol{u}_{t}\right)\right\|_{\boldsymbol{L}_{2}(\Omega)}^{2}+c_{P}\left(\|\nabla \mu\|_{\boldsymbol{L}_{2}(\Omega)}^{2}+\left\|\chi_{t}\right\|_{L_{2}(\Omega)}^{2}\right) \\
& \quad \leq \sqrt{2}\|\boldsymbol{b}\|_{\boldsymbol{L}_{2}(\Omega)} \sqrt{F}, \quad t \in[0, T] .
\end{aligned}
$$

Hence,

$$
\frac{d}{d t} \sqrt{F} \leq \frac{1}{\sqrt{2}}\|\boldsymbol{b}\|_{L_{2}(\Omega)} .
$$


Integrating (3.9) with respect to time from 0 to $t \in[0, T]$ gives

$$
\sqrt{F(t)} \leq \frac{1}{\sqrt{2}}\|\boldsymbol{b}\|_{L_{1}\left(0, t ; L_{2}(\Omega)\right)}+\sqrt{F(0)} .
$$

Further, using (3.10) in (3.8) and integrating the result with respect to time from 0 to $t \in[0, T]$ leads to

$$
\begin{aligned}
& \text { 1) } F(t)+\nu c_{*} \int_{0}^{t}\left\|\varepsilon\left(\boldsymbol{u}_{t^{\prime}}\right)\right\|_{\boldsymbol{L}_{2}(\Omega)}^{2} d t^{\prime}+c_{P} \int_{0}^{t}\left(\|\nabla \mu\|_{\boldsymbol{L}_{2}(\Omega)}^{2}+\left\|\chi_{t^{\prime}}\right\|_{L_{2}(\Omega)}^{2}\right) d t^{\prime} \\
& \leq\|\boldsymbol{b}\|_{L_{1}\left(0, t ; \boldsymbol{L}_{2}(\Omega)\right)}\left(\|\boldsymbol{b}\|_{L_{1}\left(0, t ; \boldsymbol{L}_{2}(\Omega)\right)}+\sqrt{2 F(0)}\right)+F(0) \leq 2 F(0)+\frac{3}{2}\|\boldsymbol{b}\|_{L_{1}\left(0, t ; \boldsymbol{L}_{2}(\Omega)\right)}^{2} .
\end{aligned}
$$

Now, we note that, on account of (2.6), (2.8)-(2.10) and (1.18), the following bounds hold true

$$
\begin{aligned}
F(t) & \geq \int_{\Omega}\left[\frac{1}{2}\left(\left|\boldsymbol{u}_{t}\right|^{2}+\underline{c}_{\Gamma}|\nabla \chi|^{2}\right)+\frac{1}{8} \chi^{4}+\frac{c_{*}}{4}|\varepsilon(\boldsymbol{u})|^{2}-\frac{1}{4}-\frac{c_{*}}{2} a_{3}^{2}\right] d x \\
& \geq \frac{1}{2}\left(\left\|\boldsymbol{u}_{t}\right\|_{\boldsymbol{L}_{2}(\Omega)}^{2}+\underline{c}_{\Gamma}\|\nabla \chi\|_{\boldsymbol{L}_{2}(\Omega)}^{2}\right)+c_{1}\|\boldsymbol{u}\|_{\boldsymbol{H}^{1}(\Omega)}^{2}+\frac{1}{8}\|\chi\|_{L_{4}(\Omega)}^{4}-c_{2}
\end{aligned}
$$

for $t \in[0, T]$, with constants $c_{1}, c_{2}$ defined in (3.7). From (3.11) and (3.12) we conclude (3.6). This completes the proof.

With the help of Lemma 3.2 we now derive further estimates. Firstly, let us note that from $(1.2)_{1}$ and $(1.2)_{3}$ it follows that

$$
\frac{d}{d t} \int_{\Omega} \chi d x=0
$$

which shows that the mean value of $\chi$ is preserved, i.e.

$$
f_{\Omega} \chi(t) d x=f_{\Omega} \chi_{0} d x \equiv \chi_{m} \text { for } t \in[0, T] .
$$

Since

$\int_{\Omega}\left|\chi-f_{\Omega} \chi d x^{\prime}\right|^{2} d x=\int_{\Omega}\left(\chi^{2}-2 \chi f_{\Omega} \chi d x^{\prime}+\left|f_{\Omega} \chi d x^{\prime}\right|^{2}\right) d x=\int_{\Omega} \chi^{2} d x-|\Omega|\left|f_{\Omega} \chi d x\right|^{2}$, it follows, by Poincaré inequality (1.19) and (3.14) that

$$
\|\chi\|_{L_{2}(\Omega)}^{2} \leq \int_{\Omega}\left|\chi-f_{\Omega} \chi d x^{\prime}\right|^{2} d x+|\Omega|\left|f_{\Omega} \chi d x\right|^{2} \leq d_{2}\|\nabla \chi\|_{L_{2}(\Omega)}^{2}+|\Omega| \chi_{m}^{2} .
$$

Further, on account of (3.6) and Sobolev imbedding (1.21), we infer that

$$
\sup _{t \in[0, T]}\|\chi\|_{L_{6}(\Omega)}^{2} \leq d_{4} \sup _{t \in[0, T]}\|\chi\|_{H^{1}(\Omega)}^{2} \leq d_{4}\left(\frac{4 d_{2}}{\underline{c}_{\Gamma}} \alpha_{1}+|\Omega| \chi_{m}^{2}\right) \equiv c_{3},
$$

with constant $c_{3}$ depending on $\alpha_{1}$ and $\chi_{m}$.

Next, we conclude an additional estimate on $\mu$.

Lemma 3.3. Let the assumptions of Lemma 3.2 hold. Then, for $t \in(0, T]$,

$$
\|\mu\|_{L_{2}\left(0, t ; H^{1}(\Omega)\right)}^{2} \leq c_{4}(1+t)
$$

with a positive constant $c_{4}$ depending on $\alpha_{1}$, see (3.24) below. 
Proof. From $(1.3)_{1,2}$, in view of (3.13), it follows that

$$
\int_{\Omega} \mu d x=\int_{\Omega}\left[\boldsymbol{g} \cdot \nabla \mu+\psi^{\prime}(\chi)+W_{, \chi}(\varepsilon(\boldsymbol{u}), \chi)\right] d x .
$$

Hence, using (2.5) and $(2.7)_{1}$,

$$
\left|\int_{\Omega} \mu d x\right| \leq|\boldsymbol{g}||\Omega|^{1 / 2}\|\nabla \mu\|_{\boldsymbol{L}_{2}(\Omega)}+\int_{\Omega}\left(|\chi|^{3}+|\chi|+a_{1}|\boldsymbol{\varepsilon}(\boldsymbol{u})|+a_{1}\right) d x .
$$

The second term on the right-hand side of (3.19) is bounded by

$$
\begin{aligned}
& \|\chi\|_{L_{4}(\Omega)}^{3}|\Omega|^{1 / 4}+\|\chi\|_{L_{4}(\Omega)}|\Omega|^{3 / 4}+a_{1}\|\boldsymbol{\varepsilon}(\boldsymbol{u})\|_{\boldsymbol{L}_{2}(\Omega)}|\Omega|^{1 / 2}+a_{1}|\Omega| \\
& \quad \leq\left(8 \alpha_{1}\right)^{3 / 4}|\Omega|^{1 / 4}+\left(8 \alpha_{1}\right)^{1 / 4}|\Omega|^{3 / 4}+a_{1}\left(\frac{\alpha_{1}}{c_{1}}\right)^{1 / 2}|\Omega|^{1 / 2}+a_{1}|\Omega| \equiv c_{4}^{\prime} .
\end{aligned}
$$

Hence,

$$
\left|\int_{\Omega} \mu d x\right| \leq|\boldsymbol{g}||\Omega|^{1 / 2}\|\nabla \mu\|_{\boldsymbol{L}_{2}(\Omega)}+c_{4}^{\prime} .
$$

Owing to Poincaré inequality (1.19),

$$
\|\mu\|_{L_{2}(\Omega)}^{2} \leq d_{2}\|\nabla \mu\|_{L_{2}(\Omega)}^{2}+\frac{1}{|\Omega|}\left(\int_{\Omega} \mu d x\right)^{2} .
$$

Consequently, recalling estimate (3.6), it follows from (3.21) and (3.22) that

$$
\|\mu\|_{L_{2}\left(\Omega^{t}\right)}^{2} \leq d_{2}\|\nabla \mu\|_{\boldsymbol{L}_{2}\left(\Omega^{t}\right)}^{2}+2|\boldsymbol{g}|^{2}\|\nabla \mu\|_{\boldsymbol{L}_{2}\left(\Omega^{t}\right)}^{2}+\frac{2}{|\Omega|}\left(c_{4}^{\prime}\right)^{2} t \leq c_{4}(1+t),
$$

where

$$
c_{4} \equiv \max \left\{\frac{\alpha_{1}}{c_{P}}\left(d_{2}+2|\boldsymbol{g}|^{2}\right), \frac{2}{|\Omega|}\left(c_{4}^{\prime}\right)^{2}\right\} .
$$

This concludes the proof.

Thanks to Lemma 3.3 we deduce further estimates on $\chi$.

Lemma 3.4. Let the assumptions of Lemma 3.2 hold. Then, for $t \in(0, T]$,

$$
\|\chi\|_{L_{2}\left(0, t ; H^{2}(\Omega)\right)}^{2} \leq c_{5}(1+t),
$$

with a positive constant $c_{5}$ depending on $\alpha_{1}$ and $\chi_{m}$.

Proof. Multiplying $(1.3)_{1}$ by $\Delta_{\boldsymbol{\Gamma}} \chi$ and integrating over $\Omega^{t}, t \in(0, T]$, gives

$$
\int_{0}^{t} \int_{\Omega}\left(\Delta_{\boldsymbol{\Gamma}} \chi\right)^{2} d x d t^{\prime}=\int_{0}^{t} \int_{\Omega}\left[-\mu+\boldsymbol{g} \cdot \nabla \mu+\psi^{\prime}(\chi)+W_{, \chi}(\varepsilon(\boldsymbol{u}), \chi)+\beta \chi_{t^{\prime}}\right] \Delta_{\boldsymbol{\Gamma}} \chi d x d t^{\prime} .
$$

Hence, using the Young inequality, and then (2.5), (2.7) ${ }_{1}$ together with estimates (3.6), (3.16) and (3.17), we infer that

$$
\begin{aligned}
& \left\|\Delta_{\boldsymbol{\Gamma}} \chi\right\|_{L_{2}\left(\Omega^{t}\right)}^{2} \leq 5\left[\|\mu\|_{L_{2}\left(\Omega^{t}\right)}^{2}+\|\boldsymbol{g} \cdot \nabla \mu\|_{L_{2}\left(\Omega^{t}\right)}^{2}+\left\|\psi^{\prime}(\chi)\right\|_{L_{2}\left(\Omega^{t}\right)}^{2}\right. \\
& \left.\quad+\left\|W_{, \chi}(\boldsymbol{\varepsilon}(\boldsymbol{u}), \chi)\right\|_{L_{2}\left(\Omega^{t}\right)}^{2}+\left\|\beta \chi_{t^{\prime}}\right\|_{L_{2}\left(\Omega^{t}\right)}^{2}\right] \\
& \leq 5\left[\left(1+|\boldsymbol{g}|^{2}\right)\|\mu\|_{L_{2}\left(0, t ; H^{1}(\Omega)\right)}^{2}+\sup _{t^{\prime}}\left(\|\chi\|_{L_{6}(\Omega)}^{6}\right.\right. \\
& \left.\left.\quad+\|\chi\|_{L_{6}(\Omega)}^{2}|\Omega|^{2 / 3}\right) t+2 a_{1}^{2}\left(\sup _{t^{\prime}}\|\varepsilon(\boldsymbol{u})\|_{L_{2}(\Omega)}^{2}+|\Omega|\right) t+\beta^{2}\left\|\chi_{t^{\prime}}\right\|_{L_{2}\left(\Omega^{t}\right)}^{2}\right]
\end{aligned}
$$




$$
\leq 5\left[c_{4}\left(1+|\boldsymbol{g}|^{2}\right)(1+t)+\left(c_{3}^{3}+c_{3}|\Omega|^{2 / 3}\right) t+2 a_{1}^{2}\left(\frac{\alpha_{1}}{c_{1}}+|\Omega|\right) t+\beta^{2} \frac{\alpha_{1}}{c_{P}}\right] \leq c_{5}^{\prime}(1+t),
$$

where, by definition of $c_{3}, c_{4}$ (see (3.16), (3.24)), constant $c_{5}^{\prime}$ depends on $\alpha_{1}$ and $\chi_{m}$. Finally, taking into account the inequality

$$
\|\chi\|_{H^{2}(\Omega)}^{2} \leq c\left(\left\|\Delta_{\Gamma} \chi\right\|_{L_{2}(\Omega)}^{2}+\left|\int_{\Omega} \chi d x\right|^{2}\right)
$$

which holds true due to the ellipticity property of the operator $\Delta_{\boldsymbol{\Gamma}}$, we conclude on account of (3.26) and (3.14) the bound (3.25).

Using standard duality arguments we shall estimate also time derivative $\boldsymbol{u}_{t t}$.

Lemma 3.5. Let the assumptions of Lemma 3.2 hold, and $\boldsymbol{b} \in \boldsymbol{L}_{2}\left(\Omega^{t}\right)$. Then

$$
\left\|\boldsymbol{u}_{t^{\prime} t^{\prime}}\right\|_{\left.L_{2}\left(0, t ; \boldsymbol{H}_{0}^{1}(\Omega)\right)^{\prime}\right)}^{2} \leq c_{6}(1+t),
$$

with constant $c_{6}$ depending on $\alpha_{1}$ and $\|\boldsymbol{b}\|_{\boldsymbol{L}_{2}\left(\Omega^{t}\right)}$.

Proof. We test $(1.15)_{1}$ with $\boldsymbol{\eta} \in L_{2}\left(0, T ; \boldsymbol{H}_{0}^{1}(\Omega)\right)$ and integrate over $\Omega^{t}, t \in(0, T]$. Then, using the Cauchy-Schwarz inequality and recalling estimate (3.6), it follows that

$$
\begin{aligned}
\left|\int_{\Omega^{t}} \boldsymbol{u}_{t t} \cdot \boldsymbol{\eta} d x d t^{\prime}\right|= & \left|\int_{\Omega^{t}}\left[-\left(\boldsymbol{A} \boldsymbol{\varepsilon}(\boldsymbol{u})+\nu \boldsymbol{A} \varepsilon\left(\boldsymbol{u}_{t}\right)\right) \cdot \boldsymbol{\varepsilon}(\boldsymbol{\eta})+\left(z^{\prime}(\chi) \boldsymbol{B} \nabla \chi+\boldsymbol{b}\right) \cdot \boldsymbol{\eta}\right] d x d t^{\prime}\right| \\
\leq & \left(|\boldsymbol{A}|\|\boldsymbol{\varepsilon}(\boldsymbol{u})\|\left\|_{\boldsymbol{L}_{2}\left(\Omega^{t}\right)}+\nu|\boldsymbol{A}|\right\| \varepsilon\left(\boldsymbol{u}_{t}\right) \|_{\boldsymbol{L}_{2}\left(\Omega^{t}\right)}\right)\|\varepsilon(\boldsymbol{\eta})\|_{\boldsymbol{L}_{2}\left(\Omega^{t}\right)} \\
& +\left(\mid z^{\prime}(\chi) \boldsymbol{B}\|\nabla \chi\|_{\boldsymbol{L}_{2}\left(\Omega^{t}\right)}+\|\boldsymbol{b}\|_{\boldsymbol{L}_{2}\left(\Omega^{t}\right)}\right)\|\boldsymbol{\eta}\|_{\boldsymbol{L}_{2}\left(\Omega^{t}\right)} \\
\leq & c_{6}^{\prime}\left(1+t^{1 / 2}\right)\|\boldsymbol{\eta}\|_{L_{2}\left(0, t ; \boldsymbol{H}_{0}^{1}(\Omega)\right)} \text { for all } \eta \in L_{2}\left(0, t ; \boldsymbol{H}_{0}^{1}(\Omega)\right),
\end{aligned}
$$

where constant $c_{6}^{\prime}$ depends on $|\boldsymbol{A}|, \nu,\left|z^{\prime}(\chi) \boldsymbol{B}\right| \leq a_{1},\|\boldsymbol{b}\|_{\boldsymbol{L}_{2}\left(\Omega^{t}\right)}$ and $\alpha_{1}^{1 / 2}$. This implies that

$$
\left\|\boldsymbol{u}_{t^{\prime} t^{\prime}}\right\|_{L_{2}\left(0, t ;\left(\boldsymbol{H}_{0}^{1}(\Omega)^{\prime}\right)\right.}^{2} \leq c_{6}(1+t), \quad c_{6}=2\left(c_{6}^{\prime}\right)^{2},
$$

and thereby shows (3.28).

4. Absorbing set estimate. In this section we prove an absorbing set estimate. This estimate allows firstly, to prolong the solution step by step on the infinite time interval and secondly, to conclude the existence of an absorbing set for system (1.1)-(1.3). The latter property is of interest in the long-time analysis of the problem.

LEMma 4.1. Let us define

$$
\begin{aligned}
G(t)= & \int_{\Omega}\left[\frac{1}{2}\left(\left|\boldsymbol{u}_{t}\right|^{2}+\nabla \chi \cdot \boldsymbol{\Gamma} \nabla \chi\right)+\psi(\chi)+W(\varepsilon(\boldsymbol{u}), \chi)\right. \\
& \left.+\frac{\nu c_{*} d_{1}}{2}\left(\boldsymbol{u}_{t} \cdot \boldsymbol{u}+\frac{\nu}{2} \varepsilon(\boldsymbol{u}) \cdot \boldsymbol{A} \boldsymbol{\varepsilon}(\boldsymbol{u})\right)\right] d x
\end{aligned}
$$

satisfying

$$
G(t) \geq \int_{\Omega}\left[\frac{1}{4}\left|\boldsymbol{u}_{t}\right|^{2}+\frac{1}{2} \nabla \chi \cdot \boldsymbol{\Gamma} \nabla \chi+\psi(\chi)+W(\varepsilon(\boldsymbol{u}), \chi)\right] d x .
$$

Then there exists a positive constant

$$
\delta_{*}=\min \left\{\frac{\nu c_{*} d_{1}}{8}, \frac{\underline{c}_{\Gamma}}{d^{\prime} \bar{c}_{\Gamma}}, \frac{2}{d^{\prime}}, \frac{1}{4} \sqrt{\frac{c_{*} d_{1}}{2}}, \frac{c_{*} d_{1}}{8 \nu}\right\},
$$


where $d^{\prime}=\frac{2 d_{2}}{c_{P} \underline{c}_{\Gamma}}\left(d_{2}+|\boldsymbol{g}|^{2}\right)$, such that solutions of (1.1)-(1.3) satisfy the inequality

$$
\frac{d}{d t} G+\delta_{*} G+\frac{\nu c_{*} d_{1}}{8}\left\|\boldsymbol{u}_{t}\right\|_{\boldsymbol{H}^{1}(\Omega)}^{2}+\frac{c_{P}}{4}\|\nabla \mu\|_{\boldsymbol{L}_{2}(\Omega)}^{2}+c_{P}\left\|\chi_{t}\right\|_{L_{2}(\Omega)}^{2} \leq b_{4}(t)
$$

for $t \in(0, T)$, where

$$
\begin{aligned}
b_{4}(t)= & \left(2 \nu+\frac{1}{2 \nu c_{*} d_{1}}\right)\|\boldsymbol{b}(t)\|_{\boldsymbol{L}_{2}(\Omega)}^{2}+|\Omega|\left[\frac{1}{d^{\prime}}\left(\frac{3}{2}+\frac{\underline{c}_{\Gamma}}{2 d_{2}} \chi_{m}^{2}\right)\right. \\
& \left.+\frac{1}{8} \nu c_{*}^{2} d_{1} a_{3}^{2}+\frac{27 \chi_{m}^{2}}{4 d_{1}}+\frac{\chi_{m}^{2}|\boldsymbol{g}|}{c_{P}\left(d^{\prime}\right)^{2}}\right]+|\boldsymbol{A}|^{2}\left[\nu d_{1} a_{3}^{2}+\frac{16 a_{4}^{2}|\Omega|^{2}\left(1+\chi_{m}^{2}\right)}{\nu c_{*}^{2} d_{1}\left(d^{\prime}\right)^{2}}\right] .
\end{aligned}
$$

Proof. Multiplying $(1.3)_{1}$ by $\chi$, integrating over $\Omega$ and by parts using $(1.3)_{2}$, gives

$$
\int_{\Omega} \nabla \chi \cdot \boldsymbol{\Gamma} \nabla \chi d x+\int_{\Omega}\left[\psi^{\prime}(\chi) \chi+W_{, \chi}(\varepsilon(\boldsymbol{u}), \chi) \chi\right] d x=\int_{\Omega} \mu \chi d x-\int_{\Omega} \boldsymbol{g} \cdot \nabla \mu \chi d x .
$$

Writing the first integral on the right-hand side of (4.5) in the form

$$
\int_{\Omega} \mu \chi d x=\int_{\Omega}\left(\mu-f_{\Omega} \mu d x^{\prime}\right) \chi d x+\int_{\Omega} \mu d x f_{\Omega} \chi d x
$$

and next applying the Young and the Poincare inequality (1.19) to the first term on the right-hand side of (4.6) and the mean value property (3.14) to the second one, we get

$$
\left|\int_{\Omega} \mu \chi d x\right| \leq \frac{\delta_{1}}{2}\|\chi\|_{L_{2}(\Omega)}^{2}+\frac{1}{2 \delta_{1}} d_{2}\|\nabla \mu\|_{L_{2}(\Omega)}^{2}+\left|\int_{\Omega} \mu d x\right|\left|\chi_{m}\right|, \quad \delta_{1}>0 .
$$

The second integral on the right-hand side of (4.5) is estimated with the help of the Cauchy-Schwarz and the Young inequalities to give

$$
\left|\int_{\Omega} \boldsymbol{g} \cdot \nabla \mu \chi d x\right| \leq|\boldsymbol{g}|\|\nabla \mu\|_{\boldsymbol{L}_{2}(\Omega)}\|\chi\|_{L_{2}(\Omega)} \leq \frac{\delta_{2}}{2}\|\chi\|_{L_{2}(\Omega)}^{2}+\frac{1}{2 \delta_{2}}|\boldsymbol{g}|^{2}\|\nabla \mu\|_{\boldsymbol{L}_{2}(\Omega)}^{2}, \quad \delta_{2}>0 .
$$

Using (4.7), (4.8) in (4.5) and taking into account (2.6), we arrive at

$$
\begin{aligned}
& \underline{c}_{\Gamma} \int_{\Omega}|\nabla \chi|^{2} d x+\int_{\Omega}\left[\psi^{\prime}(\chi) \chi+W_{, \chi}(\boldsymbol{\varepsilon}(\boldsymbol{u}), \chi) \chi\right] d x \\
& \quad \leq \frac{\delta_{1}+\delta_{2}}{2}\|\chi\|_{L_{2}(\Omega)}^{2}+\frac{1}{2}\left(\frac{d_{2}}{\delta_{1}}+\frac{|\boldsymbol{g}|^{2}}{\delta_{2}}\right)\|\nabla \mu\|_{\boldsymbol{L}_{2}(\Omega)}^{2}+\left|\int_{\Omega} \mu d x\right|\left|\chi_{m}\right| .
\end{aligned}
$$

Now, recalling estimate (3.15) and setting $\delta_{1}=\delta_{2}=\frac{c_{\Gamma}}{2 d_{2}}$, we deduce from (4.9) the inequality

$$
\begin{aligned}
& \frac{\underline{c_{\Gamma}}}{2} \int_{\Omega}|\nabla \chi|^{2} d x+\int_{\Omega}\left[\psi^{\prime}(\chi) \chi+W_{, \chi}(\varepsilon(\boldsymbol{u}), \chi) \chi\right] d x \\
& \quad \leq \frac{d_{2}}{\underline{c}_{\Gamma}}\left(d_{2}+|\boldsymbol{g}|^{2}\right)\|\nabla \mu\|_{\boldsymbol{L}_{2}(\Omega)}^{2}+\frac{\underline{c}_{\Gamma}|\Omega|}{2 d_{2}} \chi_{m}^{2}+\left|\int_{\Omega} \mu d x\right|\left|\chi_{m}\right| .
\end{aligned}
$$

Let us turn now to energy identity (3.2). In view of (3.1), (2.2) and structure condition (2.12) it follows that

$$
\begin{aligned}
& \left.\frac{d}{d t} \int_{\Omega}\left[\frac{1}{2}\left|\boldsymbol{u}_{t}\right|^{2}+\nabla \chi \cdot \Gamma \nabla \chi\right)+\psi(\chi)+W(\varepsilon(\boldsymbol{u}), \chi)\right] d x \\
& \quad+\nu c_{*}\left\|\varepsilon\left(\boldsymbol{u}_{t}\right)\right\|_{\boldsymbol{L}_{2}(\Omega)}^{2}+c_{P}\left(\|\nabla \mu\|_{\boldsymbol{L}_{2}(\Omega)}^{2}+\left\|\chi_{t}\right\|_{L_{2}(\Omega)}^{2}\right) \leq \int_{\Omega} \boldsymbol{b} \cdot \boldsymbol{u}_{t} d x .
\end{aligned}
$$


By the Hölder, Young and Korn (see (1.18)) inequalities,

$$
\left|\int_{\Omega} \boldsymbol{b} \cdot \boldsymbol{u}_{t} d x\right| \leq \frac{\delta_{3}}{2}\left\|\boldsymbol{u}_{t}\right\|_{\boldsymbol{L}_{2}(\Omega)}^{2}+\frac{1}{2 \delta_{3}}\|\boldsymbol{b}\|_{\boldsymbol{L}_{2}(\Omega)}^{2} \leq \frac{\delta_{3}}{2 d_{1}}\left\|\boldsymbol{\varepsilon}\left(\boldsymbol{u}_{t}\right)\right\|_{\boldsymbol{L}_{2}(\Omega)}^{2}+\frac{1}{2 \delta_{3}}\|\boldsymbol{b}\|_{\boldsymbol{L}_{2}(\Omega)}^{2} .
$$

Hence, setting $\delta_{3}=\nu c_{*} d_{1},(4.11)$ yields

$$
\begin{aligned}
& \frac{d}{d t} \int_{\Omega}\left[\frac{1}{2}\left(\left|\boldsymbol{u}_{t}\right|^{2}+\nabla \chi \cdot \boldsymbol{\Gamma} \nabla \chi\right)+\psi(\chi)+W(\varepsilon(\boldsymbol{u}), \chi)\right] d x \\
& \quad+\frac{\nu c_{*}}{2}\left\|\boldsymbol{\varepsilon}\left(\boldsymbol{u}_{t}\right)\right\|_{\boldsymbol{L}_{2}(\Omega)}^{2}+c_{P}\left(\|\nabla \mu\|_{\boldsymbol{L}_{2}(\Omega)}^{2}+\left\|\chi_{t}\right\|_{L_{2}(\Omega)}^{2}\right) \leq \frac{1}{2 \nu c_{*} d_{1}}\|\boldsymbol{b}\|_{\boldsymbol{L}_{2}(\Omega)}^{2} .
\end{aligned}
$$

Let us multiply now (4.12) by the constant

$$
d^{\prime} \equiv \frac{2 d_{2}}{c_{P} \underline{c}_{\Gamma}}\left(d_{2}+|\boldsymbol{g}|^{2}\right)>0
$$

and add to (4.10) to get

$$
\begin{aligned}
& d^{\prime} \frac{d}{d t} \int_{\Omega}\left[\frac{1}{2}\left(\left|\boldsymbol{u}_{t}\right|^{2}+\nabla \chi \cdot \Gamma \nabla \chi\right)+\psi(\chi)+W(\varepsilon(\boldsymbol{u}), \chi)\right] d x \\
& \quad+\int_{\Omega}\left[\frac{c_{\Gamma}}{2}|\nabla \chi|^{2}+\psi^{\prime}(\chi) \chi+W_{, \chi}(\boldsymbol{\varepsilon}(\boldsymbol{u}), \chi) \chi\right] d x \\
& \quad+\frac{\nu d^{\prime} c_{*}}{2}\left\|\varepsilon\left(\boldsymbol{u}_{t}\right)\right\|_{\boldsymbol{L}_{2}(\Omega)}^{2}+\frac{d^{\prime} c_{P}}{2}\|\nabla \mu\|_{\boldsymbol{L}_{2}(\Omega)}^{2}+d^{\prime} c_{P}\left\|\chi_{t}\right\|_{L_{2}(\Omega)}^{2} \\
& \leq \frac{c_{\Gamma}|\Omega|}{2 d_{2}} \chi_{m}^{2}+\left|\int_{\Omega} \mu d x\right|\left|\chi_{m}\right|+\frac{d^{\prime}}{2 \nu c_{*} d_{1}}\|\boldsymbol{b}\|_{\boldsymbol{L}_{2}(\Omega)}^{2} .
\end{aligned}
$$

Noting that $\int_{\Omega} \psi^{\prime}(\chi) \chi d x=\int_{\Omega}\left(\chi^{4}-\chi^{2}\right) d x$ and

$$
\int_{\Omega} \psi(\chi) d x=\frac{1}{4} \int_{\Omega}\left(\chi^{4}+1-2 \chi^{2}\right) d x=\frac{1}{4} \int_{\Omega}\left(\chi^{4}-\chi^{2}\right) d x+\frac{1}{4} \int_{\Omega}\left(1-\chi^{2}\right) d x,
$$

we have

$$
\int_{\Omega} \psi(\chi) d x=\frac{1}{4} \int_{\Omega} \psi^{\prime}(\chi) \chi d x+\frac{1}{4} \int_{\Omega}\left(1-\chi^{2}\right) d x \leq \frac{1}{4} \int_{\Omega} \psi^{\prime}(\chi) \chi d x+\frac{|\Omega|}{4} .
$$

Further, by assumption (2.6),

$$
\frac{\underline{c}_{\Gamma}}{2} \int_{\Omega}|\nabla \chi|^{2} d x \geq \frac{c_{\Gamma}}{\bar{c}_{\Gamma}} \int_{\Omega} \frac{1}{2} \nabla \chi \cdot \Gamma \nabla \chi d x .
$$

Using (4.15) and (4.16) in (4.14), and dividing the result by $d^{\prime}>0$ gives

$$
\begin{aligned}
& \frac{d}{d t} \int_{\Omega}\left[\frac{1}{2}\left(\left|\boldsymbol{u}_{t}\right|^{2}+\nabla \chi \cdot \Gamma \nabla \chi\right)+\psi(\chi)+W(\varepsilon(\boldsymbol{u}), \chi)\right] d x \\
& \quad+\frac{1}{d^{\prime}} \int_{\Omega}\left[\frac{\underline{c}_{\Gamma}}{2 \bar{c}_{\Gamma}} \nabla \chi \cdot \boldsymbol{\Gamma} \nabla \chi+4 \psi(\chi)+W_{, \chi}(\boldsymbol{\varepsilon}(\boldsymbol{u}), \chi) \chi\right] d x \\
& \quad+\frac{\nu c_{*}}{2}\left\|\varepsilon\left(\boldsymbol{u}_{t}\right)\right\|_{\boldsymbol{L}_{2}(\Omega)}^{2}+\frac{c_{P}}{2}\|\nabla \mu\|_{\boldsymbol{L}_{2}(\Omega)}^{2}+c_{P}\left\|\chi_{t}\right\|_{L_{2}(\Omega)}^{2} \leq \frac{\left|\chi_{m}\right|}{d^{\prime}}\left|\int_{\Omega} \mu d x\right|+b_{1}^{2},
\end{aligned}
$$

where

$$
b_{1}^{2}=\frac{1}{2 \nu c_{*} d_{1}}\|\boldsymbol{b}\|_{\boldsymbol{L}_{2}(\Omega)}^{2}+\frac{|\Omega|}{d^{\prime}}\left(1+\frac{\underline{c}_{\Gamma}}{2 d_{2}} \chi_{m}^{2}\right)
$$


Let us turn now to elasticity system (1.1). Multiplying (1.1) $)_{1}$ by $\boldsymbol{u}$ and integrating over $\Omega$ yields

$$
\begin{aligned}
\frac{d}{d t} \int_{\Omega} \boldsymbol{u}_{t} \cdot \boldsymbol{u} d x+\int_{\Omega} W_{, \varepsilon}(\varepsilon(\boldsymbol{u}), \chi) \cdot \boldsymbol{\varepsilon}(\boldsymbol{u}) d x & +\nu \int_{\Omega} \boldsymbol{\varepsilon}(\boldsymbol{u}) \cdot \boldsymbol{A} \varepsilon\left(\boldsymbol{u}_{t}\right) d x \\
& =\int_{\Omega} \boldsymbol{b} \cdot \boldsymbol{u} d x+\int_{\Omega}\left|\boldsymbol{u}_{t}\right|^{2} d x .
\end{aligned}
$$

Using that

$$
W_{, \varepsilon}(\varepsilon(\boldsymbol{u}), \chi) \cdot \varepsilon(\boldsymbol{u})=\varepsilon(\boldsymbol{u}) \cdot \boldsymbol{A}(\varepsilon(\boldsymbol{u})-\bar{\varepsilon}(\chi))=2 W(\varepsilon(\boldsymbol{u}), \chi)+\bar{\varepsilon}(\chi) \cdot \boldsymbol{A}(\varepsilon(\boldsymbol{u})-\bar{\varepsilon}(\chi)),
$$

we obtain from (4.18) the equality

$$
\begin{aligned}
& \frac{d}{d t} \int_{\Omega}\left(\boldsymbol{u}_{t} \cdot \boldsymbol{u}+\frac{\nu}{2} \varepsilon(\boldsymbol{u}) \cdot \boldsymbol{A} \varepsilon(\boldsymbol{u})\right) d x+2 \int_{\Omega} W(\varepsilon(\boldsymbol{u}), \chi) d x \\
& =-\int_{\Omega} \overline{\boldsymbol{\varepsilon}}(\chi) \cdot \boldsymbol{A}(\varepsilon(\boldsymbol{u})-\overline{\boldsymbol{\varepsilon}}(\chi)) d x+\int_{\Omega} \boldsymbol{b} \cdot \boldsymbol{u} d x+\int_{\Omega}\left|\boldsymbol{u}_{t}\right|^{2} d x
\end{aligned}
$$

We use now the following inequalities (see (2.8), (2.10), (1.18))

$$
\begin{aligned}
& \int_{\Omega} W(\boldsymbol{\varepsilon}(\boldsymbol{u}), \chi) d x \geq \frac{c_{*}}{2} \int_{\Omega}|\boldsymbol{\varepsilon}(\boldsymbol{u})-\bar{\varepsilon}(\chi)|^{2} d x \\
& \int_{\Omega} W(\varepsilon(\boldsymbol{u}), \chi) d x \geq \frac{c_{*}}{2} \int_{\Omega}\left(\frac{1}{2}|\varepsilon(\boldsymbol{u})|^{2}-|\overline{\boldsymbol{\varepsilon}}(\chi)|^{2}\right) d x \geq \frac{c_{*}}{4} d_{1}\|\boldsymbol{u}\|_{\boldsymbol{H}^{1}(\Omega)}^{2}-\frac{c_{*}}{2} a_{3}^{2}|\Omega|
\end{aligned}
$$

and

$$
\begin{aligned}
& \left|\int_{\Omega} \overline{\boldsymbol{\varepsilon}}(\chi) \cdot \boldsymbol{A}(\boldsymbol{\varepsilon}(\boldsymbol{u})-\overline{\boldsymbol{\varepsilon}}(\chi)) d x\right| \leq|\overline{\boldsymbol{\varepsilon}}(\chi) \| \boldsymbol{A}| \int_{\Omega}|\boldsymbol{\varepsilon}(\boldsymbol{u})-\overline{\boldsymbol{\varepsilon}}(\chi)| d x \\
& \leq \frac{\delta_{4}}{2} \int_{\Omega}|\boldsymbol{\varepsilon}(\boldsymbol{u})-\overline{\boldsymbol{\varepsilon}}(\chi)|^{2} d x+\frac{1}{2 \delta_{4}} a_{3}^{2}|\boldsymbol{A}|^{2}, \quad \delta_{4}>0, \\
& \left|\int_{\Omega} \boldsymbol{b} \cdot \boldsymbol{u} d x\right| \leq \frac{\delta_{5}}{2}\|\boldsymbol{u}\|_{\boldsymbol{L}_{2}(\Omega)}^{2}+\frac{1}{2 \delta_{5}}\|\boldsymbol{b}\|_{\boldsymbol{L}_{2}(\Omega)}^{2}, \quad \delta_{5}>0 .
\end{aligned}
$$

On account of (4.20), (4.21) we infer from (4.19) the inequality

$$
\begin{aligned}
& \frac{d}{d t} \int_{\Omega}\left(\boldsymbol{u}_{t} \cdot \boldsymbol{u}+\frac{\nu}{2} \boldsymbol{\varepsilon}(\boldsymbol{u}) \cdot \boldsymbol{A} \boldsymbol{\varepsilon}(\boldsymbol{u})\right) d x+\frac{1}{2} \frac{c_{*}}{2} \int_{\Omega}|\varepsilon(\boldsymbol{u})-\overline{\boldsymbol{\varepsilon}}(\chi)|^{2} d x \\
& \quad+\frac{1}{2} \frac{c_{*} d_{1}}{4}\|\boldsymbol{u}\|_{\boldsymbol{H}^{1}(\Omega)}^{2}+\int_{\Omega} W(\boldsymbol{\varepsilon}(\boldsymbol{u}), \chi) d x \\
& \leq \frac{\delta_{4}}{2} \int_{\Omega}|\boldsymbol{\varepsilon}(\boldsymbol{u})-\overline{\boldsymbol{\varepsilon}}(\chi)|^{2} d x+\frac{1}{2 \delta_{4}} a_{3}^{2}|\boldsymbol{A}|^{2}+\frac{\delta_{5}}{2}\|\boldsymbol{u}\|_{\boldsymbol{L}_{2}(\Omega)}^{2} \\
& \quad+\frac{1}{2 \delta_{5}}\|\boldsymbol{b}\|_{\boldsymbol{L}_{2}(\Omega)}^{2}+\frac{1}{2} \frac{c_{*} a_{3}^{2}}{2}|\Omega|+\left\|\boldsymbol{u}_{t}\right\|_{\boldsymbol{L}_{2}(\Omega)}^{2} .
\end{aligned}
$$

Assuming that $\delta_{4}=c_{*} / 4, \delta_{5}=c_{*} d_{1} / 8,(4.22)$ leads to

$$
\begin{aligned}
\frac{d}{d t} \int_{\Omega}\left(\boldsymbol{u}_{t} \cdot \boldsymbol{u}+\frac{\nu}{2} \varepsilon(\boldsymbol{u}) \cdot \boldsymbol{A} \varepsilon(\boldsymbol{u})\right) d x+\frac{c_{*}}{8} \int_{\Omega}|\boldsymbol{\varepsilon}(\boldsymbol{u})-\overline{\boldsymbol{\varepsilon}}(\chi)|^{2} d x \\
+\frac{c_{*} d_{1}}{16}\|\boldsymbol{u}\|_{\boldsymbol{H}^{1}(\Omega)}^{2}+\int_{\Omega} W(\varepsilon(\boldsymbol{u}), \chi) d x \leq\left\|\boldsymbol{u}_{t}\right\|_{\boldsymbol{L}_{2}(\Omega)}^{2}+b_{2}^{2},
\end{aligned}
$$


where

$$
b_{2}^{2}=\frac{2}{c_{*}} a_{3}^{2}|\boldsymbol{A}|^{2}+\frac{1}{4} c_{*} a_{3}^{2}|\Omega|+\frac{4}{c_{*} d_{1}}\|\boldsymbol{b}\|_{\boldsymbol{L}_{2}(\Omega)}^{2} .
$$

Now we multiply (4.23) by a constant $\delta_{6}>0$ (to be chosen later on) and add to (4.17) to get after using Korn's inequality (1.18)

$$
\begin{aligned}
& \frac{d}{d t} \int_{\Omega}\left[\frac{1}{2}\left(\left|\boldsymbol{u}_{t}\right|^{2}+\nabla \chi \cdot \boldsymbol{\Gamma} \nabla \chi\right)+\psi(\chi)+W(\varepsilon(\boldsymbol{u}), \chi)\right. \\
& \left.\quad+\delta_{6}\left(\boldsymbol{u}_{t} \cdot \boldsymbol{u}+\frac{\nu}{2} \varepsilon(\boldsymbol{u}) \cdot \boldsymbol{A} \varepsilon(\boldsymbol{u})\right)\right] d x \\
& \quad+\frac{1}{d^{\prime}} \int_{\Omega}\left[\frac{\underline{c}_{\Gamma}}{2 \bar{c}_{\Gamma}} \nabla \chi \cdot \boldsymbol{\Gamma} \nabla \chi+4 \psi(\chi)+\frac{\delta_{6} c_{*} d^{\prime}}{8}|\varepsilon(\boldsymbol{u})-\overline{\boldsymbol{\varepsilon}}(\chi)|^{2}\right. \\
& \left.\quad+\frac{\delta_{6} c_{*} d_{1} d^{\prime}}{16}\left(|\nabla \boldsymbol{u}|^{2}+|\boldsymbol{u}|^{2}\right)+\delta_{6} d^{\prime} W(\varepsilon(\boldsymbol{u}), \chi)\right] d x \\
& \quad+\frac{\nu c_{*} d_{1}}{2}\left\|\boldsymbol{u}_{t}\right\|_{\boldsymbol{H}^{1}(\Omega)}^{2}+\frac{c_{P}}{2}\|\nabla \mu\|_{\boldsymbol{L}_{2}(\Omega)}^{2}+c_{P}\left\|\chi_{t}\right\|_{L_{2}(\Omega)}^{2} \\
& \leq \frac{1}{d^{\prime}}\left|\int_{\Omega} W_{, \chi}(\boldsymbol{\varepsilon}(\boldsymbol{u}), \chi) \chi d x\right|+\frac{\left|\chi_{m}\right|}{d^{\prime}}\left|\int_{\Omega} \mu d x\right|+\delta_{6}\left\|\boldsymbol{u}_{t}\right\|_{\boldsymbol{L}_{2}(\Omega)}^{2}+\delta_{6} b_{2}^{2}+b_{1}^{2}
\end{aligned}
$$

We estimate the first two integrals on the right-hand side of (4.24). In view of $(2.10)_{2}$,

$$
\begin{gathered}
\frac{1}{d^{\prime}}\left|\int_{\Omega} W_{, \chi}(\varepsilon(\boldsymbol{u}), \chi) \chi d x\right| \leq \frac{1}{d^{\prime}} a_{4}|\boldsymbol{A}| \int_{\Omega}|\varepsilon(\boldsymbol{u})-\bar{\varepsilon}(\chi)| d x \\
\leq \frac{1}{d^{\prime}} a_{4}\left|\boldsymbol{A}\left\|\left.\Omega\right|^{1 / 2}\right\| \varepsilon(\boldsymbol{u})-\overline{\boldsymbol{\varepsilon}}(\chi) \|_{\boldsymbol{L}_{2}(\Omega)} .\right.
\end{gathered}
$$

Recalling the identity

$$
\int_{\Omega} \mu d x=\int_{\Omega}\left[\boldsymbol{g} \cdot \nabla \mu+\psi^{\prime}(\chi)+W_{, \chi}(\varepsilon(\boldsymbol{u}), \chi)\right] d x
$$

we have

$$
\left|\int_{\Omega} \mu d x\right| \leq|\boldsymbol{g}||\Omega|^{1 / 2}\|\nabla \mu\|_{\boldsymbol{L}_{2}(\Omega)}+\left|\int_{\Omega} \psi^{\prime}(\chi) d x\right|+a_{4}\left|\boldsymbol{A}\left\|\left.\Omega\right|^{1 / 2}\right\| \varepsilon(\boldsymbol{u})-\bar{\varepsilon}(\chi) \|_{\boldsymbol{L}_{2}(\Omega)} .\right.
$$

Further, on account of (3.14),

$$
\left|\int_{\Omega} \psi^{\prime}(\chi) d x\right|=\left|\int_{\Omega}\left(\chi^{3}-\chi\right) d x\right|=\left|\int_{\Omega} \chi^{3} d x-\right| \Omega\left|\chi_{m}\right| \leq \int_{\Omega}|\chi|^{3} d x+|\Omega|\left|\chi_{m}\right| .
$$

Hence, by the Young inequality,

$$
\int_{\Omega}|\chi|^{3} d x \leq \frac{3}{4} \delta_{7}^{4 / 3} \int_{\Omega} \chi^{4} d x+\frac{1}{4 \delta_{7}^{4}}|\Omega|,
$$

and the fact that $($ see $(2.9)) \chi^{4} \leq 8 \psi(\chi)+2$, we deduce that

Consequently

$$
\left|\int_{\Omega} \psi^{\prime}(\chi) d x\right| \leq 6 \delta_{7}^{4 / 3} \int_{\Omega} \psi(\chi) d x+\left(\frac{3}{2} \delta_{7}^{4 / 3}+\frac{1}{4 \delta_{7}^{4}}\right)|\Omega| .
$$

$$
\begin{aligned}
\left|\int_{\Omega} \mu d x\right| \leq & |\boldsymbol{g}||\Omega|^{1 / 2}\|\nabla \mu\|_{\boldsymbol{L}_{2}(\Omega)}+a_{4}\left|\boldsymbol{A}\left\|\left.\Omega\right|^{1 / 2}\right\| \boldsymbol{\varepsilon}(\boldsymbol{u})-\bar{\varepsilon}(\chi) \|_{\boldsymbol{L}_{2}(\Omega)}\right. \\
& +6 \delta_{7}^{4 / 3} \int_{\Omega} \psi(\chi) d x+\left(\frac{3}{2} \delta_{7}^{4 / 3}+\frac{1}{4 \delta_{7}^{4}}\right)|\Omega| .
\end{aligned}
$$


Using (4.25) and (4.26) in (4.24), and then choosing constants $\delta_{6}, \delta_{7}$ such that

$$
\delta_{6} \leq \frac{\nu c_{*} d_{1}}{4}, \quad \frac{\left|\chi_{m}\right|}{d^{\prime}} 6 \delta_{7}^{4 / 3}=\frac{2}{d^{\prime}} \quad \text { so } \quad \delta_{7}=\frac{1}{\left(3\left|\chi_{m}\right|\right)^{3 / 4}},
$$

we obtain

$$
\begin{aligned}
& \frac{d}{d t} \int_{\Omega}\left[\frac{1}{2}\left(\left|\boldsymbol{u}_{t}\right|^{2}+\nabla \chi \cdot \boldsymbol{\Gamma} \nabla \chi\right)+\psi(\chi)+W(\varepsilon(\boldsymbol{u}), \chi)\right. \\
& \left.\quad+\delta_{6}\left(\boldsymbol{u}_{t} \cdot \boldsymbol{u}+\frac{\nu}{2} \varepsilon(\boldsymbol{u}) \cdot \boldsymbol{A} \varepsilon(\boldsymbol{u})\right)\right] d x+\frac{1}{d^{\prime}} \int_{\Omega}\left[\frac{c_{\Gamma}}{2 \bar{c}_{\Gamma}} \nabla \chi \cdot \Gamma \nabla \chi+2 \psi(\chi)\right. \\
& \left.\quad+\frac{\delta_{6} c_{*} d^{\prime}}{8}|\varepsilon(\boldsymbol{u})-\bar{\varepsilon}(\chi)|^{2}+\frac{\delta_{6} c_{*} d_{1} d^{\prime}}{16}\left(|\nabla \boldsymbol{u}|^{2}+|\boldsymbol{u}|^{2}\right)+\delta_{6} d^{\prime} W(\varepsilon(\boldsymbol{u}), \chi)\right] d x \\
& \quad+\frac{\nu c_{*} d_{1}}{4}\left\|\boldsymbol{u}_{t}\right\|_{\boldsymbol{H}^{1}(\Omega)}^{2}+\frac{c_{P}}{2}\|\nabla \mu\|_{\boldsymbol{L}_{2}(\Omega)}^{2}+c_{P}\left\|\chi_{t}\right\|_{L_{2}(\Omega)}^{2} \\
& \leq \frac{1}{d^{\prime}} a_{4}\left|\boldsymbol{A}\left\|\left.\Omega\right|^{1 / 2}\left(\left|\chi_{m}\right|+1\right)\right\| \boldsymbol{\varepsilon}(\boldsymbol{u})-\overline{\boldsymbol{\varepsilon}}(\chi)\left\|_{\boldsymbol{L}_{2}(\Omega)}+\frac{\left|\chi_{m}\right|}{d^{\prime}}\left|\boldsymbol{g}\left\|\left.\Omega\right|^{1 / 2}\right\| \nabla \mu \|_{\boldsymbol{L}_{2}(\Omega)}+b_{3}^{2},\right.\right.\right.
\end{aligned}
$$

where

$$
b_{3}^{2}=b_{1}^{2}+\delta_{6} b_{2}^{2}+\frac{2+27 \chi_{m}^{4}}{4 d^{\prime}}|\Omega|
$$

Estimating

$$
\begin{aligned}
\frac{1}{d^{\prime}} a_{4}\left|\boldsymbol{A}\left\|\left.\Omega\right|^{1 / 2}\left(\chi_{m}+1\right)\right\| \varepsilon(\boldsymbol{u})-\bar{\varepsilon}(\chi) \|_{\boldsymbol{L}_{2}(\Omega)}\right. & \\
& \leq \frac{\delta_{8}}{2}\|\boldsymbol{\varepsilon}(\boldsymbol{u})-\bar{\varepsilon}(\chi)\|_{\boldsymbol{L}_{2}(\Omega)}^{2}+\frac{1}{\delta_{8}} \frac{a_{4}^{2}|\boldsymbol{A}|^{2}|\Omega|\left(\chi_{m}^{2}+1\right)}{\left(d^{\prime}\right)^{2}} \\
\frac{\left|\chi_{m}\right|}{d^{\prime}}\left|\boldsymbol{g}\left\|\left.\Omega\right|^{1 / 2}\right\| \nabla \mu \|_{\boldsymbol{L}_{2}(\Omega)}\right. & \leq \frac{\delta_{9}}{2}\|\nabla \mu\|_{\boldsymbol{L}_{2}(\Omega)}^{2}+\frac{1}{2 \delta_{9}} \frac{\chi_{m}^{2}|\boldsymbol{g}|^{2}|\Omega|}{\left(d^{\prime}\right)^{2}}
\end{aligned}
$$

and choosing constants $\delta_{8}, \delta_{9}$ so that

$$
\delta_{8}=\frac{\delta_{6} c_{*}}{8}, \quad \delta_{9}=\frac{c_{P}}{2},
$$

inequality (4.28) is reduced to the form

$$
\begin{aligned}
& \frac{d}{d t} \int_{\Omega}\left[\frac{1}{2}\left(\left|\boldsymbol{u}_{t}\right|^{2}+\nabla \chi \cdot \boldsymbol{\Gamma} \nabla \chi\right)+\psi(\chi)+W(\varepsilon(\boldsymbol{u}), \chi)\right. \\
& \left.\quad+\delta_{6}\left(\boldsymbol{u}_{t} \cdot \boldsymbol{u}+\frac{\nu}{2} \boldsymbol{\varepsilon}(\boldsymbol{u}) \cdot \boldsymbol{A} \varepsilon(\boldsymbol{u})\right)\right] d x \\
& \quad+\frac{1}{d^{\prime}} \int_{\Omega}\left[\frac{c_{\Gamma}}{2 \bar{c}_{\Gamma}} \nabla \chi \cdot \Gamma \nabla \chi+2 \psi(\chi)+\frac{\delta_{6} c_{*} d^{\prime}}{16}|\boldsymbol{\varepsilon}(\boldsymbol{u})-\varepsilon(\chi)|^{2}\right. \\
& \left.\quad+\frac{\delta_{6} c_{*} d_{1} d^{\prime}}{16}\left(|\nabla \boldsymbol{u}|^{2}+|\boldsymbol{u}|^{2}\right)+\delta_{6} d^{\prime} W(\boldsymbol{\varepsilon}(\boldsymbol{u}), \chi)\right] d x \\
& \quad+\frac{\nu c_{*} d_{1}}{4}\left\|\boldsymbol{u}_{t}\right\|_{\boldsymbol{H}^{1}(\Omega)}^{2}+\frac{c_{P}}{4}\|\nabla \mu\|_{\boldsymbol{L}_{2}(\Omega)}^{2}+c_{P}\left\|\chi_{t}\right\|_{L_{2}(\Omega)}^{2} \leq b_{4}^{2},
\end{aligned}
$$

where

$$
b_{4}^{2}=b_{3}^{2}+\frac{8}{\delta_{6} c_{*}} \frac{a_{4}^{2}|\boldsymbol{A}|^{2}|\Omega|^{2}\left(\chi_{m}^{2}+1\right)}{\left(d^{\prime}\right)^{2}}+\frac{\chi_{m}^{2}|\boldsymbol{g}|^{2}|\Omega|}{c_{P}\left(d^{\prime}\right)^{2}} .
$$


Let

$$
\begin{aligned}
G(t)= & \int_{\Omega}\left[\frac{1}{2}\left(\left|\boldsymbol{u}_{t}\right|^{2}+\nabla \chi \cdot \boldsymbol{\Gamma} \nabla \chi\right)+\psi(\chi)+W(\varepsilon(\boldsymbol{u}), \chi)\right. \\
& \left.+\delta_{6}\left(\boldsymbol{u}_{t} \cdot \boldsymbol{u}+\frac{\nu}{2} \boldsymbol{\varepsilon}(\boldsymbol{u}) \cdot \boldsymbol{A} \varepsilon(\boldsymbol{u})\right)\right] d x
\end{aligned}
$$

We choose constant $\delta_{10}>0$ so that

$$
\begin{aligned}
& \delta_{10} G(t) \leq \frac{1}{d^{\prime}} \int_{\Omega}\left[\frac{\underline{c}_{\Gamma}}{2 \bar{c}_{\Gamma}} \nabla \chi \cdot \Gamma \nabla \chi+2 \psi(\chi)+\frac{\delta_{6} c_{*} d^{\prime}}{16}|\varepsilon(\boldsymbol{u})-\bar{\varepsilon}(\chi)|^{2}\right. \\
& \left.+\frac{\delta_{6} c_{*} d_{1} d^{\prime}}{16}\left(|\boldsymbol{u}|^{2}+|\nabla \boldsymbol{u}|^{2}\right)+\delta_{6} d^{\prime} W(\varepsilon(\boldsymbol{u}), \chi)+\frac{\nu c_{*} d_{1} d^{\prime}}{8}\left(\left|\boldsymbol{u}_{t}\right|^{2}+\left|\nabla \boldsymbol{u}_{t}\right|^{2}\right)\right] d x .
\end{aligned}
$$

This can be satisfied under the following conditions:

$$
\begin{array}{cc}
\frac{\delta_{10}}{2}\left|\boldsymbol{u}_{t}\right|^{2} \leq \frac{\nu c_{*} d_{1}}{16}\left|\boldsymbol{u}_{t}\right|^{2}, & \text { so } \quad \delta_{10} \leq \frac{\nu c_{*} d_{1}}{8}, \\
\frac{\delta_{10}}{2} \nabla \chi \cdot \Gamma \nabla \chi \leq \frac{1}{d^{\prime}} \frac{\underline{c}_{\Gamma}}{2 \bar{c}_{\Gamma},} & \text { so } \quad \delta_{10} \leq \frac{\underline{c}_{\Gamma}}{d^{\prime} \bar{c}_{\Gamma}}, \\
\delta_{10} \psi(\chi) \leq \frac{2}{d^{\prime}} \psi(\chi), & \text { so } \quad \delta_{10} \leq \frac{2}{d^{\prime}}, \\
\delta_{10} W(\varepsilon(\boldsymbol{u}), \chi) \leq \delta_{6} W(\varepsilon(\boldsymbol{u}), \chi), & \text { so } \quad \delta_{10} \leq \delta_{6}, \\
\delta_{10} \delta_{6} \boldsymbol{u}_{t} \cdot \boldsymbol{u} \leq \delta_{10} \delta_{6}\left(\frac{\delta_{11}}{2}\left|\boldsymbol{u}_{t}\right|^{2}+\frac{1}{2 \delta_{11}}|\boldsymbol{u}|^{2}\right) \leq \frac{\nu c_{*} d_{1}}{16}\left|\boldsymbol{u}_{t}\right|^{2}+\frac{\delta_{6} c_{*} d_{1}}{16}|\boldsymbol{u}|^{2},
\end{array}
$$

so e.g.

$$
\begin{aligned}
& \delta_{11}=\frac{\nu c_{*} d_{1}}{8 \delta_{10} \delta_{6}} \quad \text { and } \quad \delta_{10}^{2} \leq \frac{\nu c_{*}^{2} d_{1}^{2}}{64 \delta_{6}} \\
& \delta_{10} \delta_{6} \frac{\nu}{2} \boldsymbol{\varepsilon}(\boldsymbol{u}) \cdot \boldsymbol{A} \boldsymbol{\varepsilon}(\boldsymbol{u}) \leq \frac{\delta_{6} c_{*} d_{1}}{16}\left(|\boldsymbol{u}|^{2}+|\nabla \boldsymbol{u}|^{2}\right)
\end{aligned}
$$

SO

$$
\delta_{10} \leq \frac{c_{*} d_{1}}{8 \nu}
$$

Consequently, choosing

$$
\delta_{10}=\min \left\{\frac{\nu c_{*} d_{1}}{8}, \frac{c_{\Gamma}}{d^{\prime} \bar{c}_{\Gamma}}, \frac{2}{d^{\prime}}, \delta_{6}, \frac{c_{*} d_{1}}{8} \sqrt{\frac{\nu}{\delta_{6}}}, \frac{c_{*} d_{1}}{8 \nu}\right\},
$$

inequality (4.29) yields

$$
\frac{d}{d t} G(t)+\delta_{10} G(t)+\frac{\nu c_{*} d_{1}}{8}\left\|\boldsymbol{u}_{t}\right\|_{\boldsymbol{H}^{1}(\Omega)}^{2}+\frac{c_{P}}{4}\|\nabla \mu\|_{\boldsymbol{L}_{2}(\Omega)}^{2}+c_{P}\left\|\chi_{t}\right\|_{L_{2}(\Omega)}^{2} \leq b_{4}^{2} .
$$

Finally, we choose constant $\delta_{6}>0$ so that

$$
G(t) \geq \int_{\Omega}\left[\frac{1}{4}\left|\boldsymbol{u}_{t}\right|^{2}+\frac{1}{2} \nabla \chi \cdot \Gamma \nabla \chi+\psi(\chi)+W(\varepsilon(\boldsymbol{u}), \chi)\right] d x .
$$

In fact, taking into account that (see (1.18), (2.2))

$$
\int_{\Omega} \boldsymbol{\varepsilon}(\boldsymbol{u}) \cdot \boldsymbol{A} \boldsymbol{\varepsilon}(\boldsymbol{u}) d x \geq c_{*} d_{1} \int_{\Omega}|\boldsymbol{u}|^{2} d x
$$


we have

$$
\begin{aligned}
G(t) \geq & \int_{\Omega}\left[\frac{1}{2}\left|\boldsymbol{u}_{t}\right|^{2}+\frac{1}{2} \nabla \chi \cdot \Gamma \nabla \chi+\psi(\chi)+W(\varepsilon(\boldsymbol{u}), \chi)\right. \\
& \left.-\delta_{6}\left(\frac{\delta}{2}\left|\boldsymbol{u}_{t}\right|^{2}+\frac{1}{2 \delta}|\boldsymbol{u}|^{2}\right)+\delta_{6} \frac{\nu}{2} c_{*} d_{1}|\boldsymbol{u}|^{2}\right] d x
\end{aligned}
$$

where $\delta>0$. Hence, choosing

$$
\frac{\delta_{6} \delta}{2}=\frac{1}{4} \quad \text { and } \quad \frac{\delta_{6}}{2 \delta}=\frac{\delta_{6} \nu c_{*} d_{1}}{2}
$$

that is, $\delta=\frac{1}{\nu c_{*} d_{1}}$ and $\delta_{6}=\frac{\nu c_{*} d_{1}}{2}$, we ensure the bound (4.34).

For $\delta_{6}=\frac{\nu c_{*} d_{1}}{2}$, condition (4.32) becomes

$$
\delta_{10}=\min \left\{\frac{\nu c_{*} d_{1}}{8}, \frac{\underline{c}_{\Gamma}}{d^{\prime} \bar{c}_{\Gamma}}, \frac{2}{d^{\prime}}, \frac{1}{4} \sqrt{\frac{c_{*} d_{1}}{2}}, \frac{c_{*} d_{1}}{8 \nu}\right\} .
$$

Thereby the assertion of lemma is proved.

\section{Existence of weak solutions}

Proof of Theorem 2.1. The proof modifies slightly the proofs of Theorems 3.1 and 3.2 from [BarPaw05].

Step 1. The Faedo-Galerkin approximation. We introduce the following eigenvalue problems

$$
-\boldsymbol{Q} \boldsymbol{v}_{j}=\lambda_{j}^{(1)} \boldsymbol{v}_{j} \quad \text { in } \Omega, \quad \boldsymbol{v}_{j}=\mathbf{0} \quad \text { on } S, j \in \mathbb{N},
$$

where $\boldsymbol{Q}$ is the elliptic operator defined by (1.11). Moreover,

$$
\begin{gathered}
-\Delta_{M} w_{j}=\lambda_{j}^{(2)} w_{j} \quad \text { in } \Omega, \\
-\Delta_{\Gamma} z_{j}=\lambda_{j}^{(3)} z_{j} \quad \text { in } \Omega,
\end{gathered}
$$

with boundary conditions

$$
\begin{array}{ll}
\boldsymbol{n} \cdot\left(\boldsymbol{M} \nabla w_{j}+\boldsymbol{h} z_{j}\right)=0 & \text { on } S, \\
\boldsymbol{n} \cdot \boldsymbol{\Gamma} \nabla z_{j}=0 & \text { on } S .
\end{array}
$$

The sets $\left\{\boldsymbol{v}_{j}\right\},\left\{w_{j}\right\}$ and $\left\{z_{j}\right\}, j \in \mathbb{N}$, form bases in $\boldsymbol{H}_{0}^{1}(\Omega), H^{1}(\Omega)$, and $H^{1}(\Omega)$, respectively. Define

$$
\boldsymbol{V}_{m}=\operatorname{span}\left\{\boldsymbol{v}_{1}, \ldots, \boldsymbol{v}_{m}\right\}, \quad W_{m}=\operatorname{span}\left\{w_{1}, \ldots, w_{m}\right\}, \quad Z_{m}=\operatorname{span}\left\{z_{1}, \ldots, z_{m}\right\} .
$$

To construct the Faedo-Galerkin approximation for (1.1)-(1.3) we consider the following regularization of (1.1)-(1.3): 


$$
\begin{array}{ll}
\boldsymbol{u}_{t t}^{\gamma}-\nabla \cdot\left[W_{, \varepsilon}\left(\varepsilon\left(\boldsymbol{u}^{\gamma}\right), \chi^{\gamma}\right)+\nu \boldsymbol{A} \varepsilon\left(\boldsymbol{u}_{t}^{\gamma}\right)\right]=\boldsymbol{b} & \text { in } \Omega^{T}, \\
\left.\boldsymbol{u}^{\gamma}\right|_{t=0}=\boldsymbol{u}_{0},\left.\quad \boldsymbol{u}_{t}^{\gamma}\right|_{t=0}=\boldsymbol{u}_{1} & \text { in } \Omega, \\
\boldsymbol{u}^{\gamma}=\mathbf{0} & \text { on } S^{T}, \\
\gamma \mu_{t}^{\gamma}+\chi_{t}^{\gamma}-\nabla \cdot\left(\boldsymbol{M} \nabla \mu^{\gamma}+\boldsymbol{h} \chi_{t}^{\gamma}\right)=0 & \text { in } \Omega^{T}, \\
\left.\chi^{\gamma}\right|_{t=0}=\chi_{0} & \text { in } \Omega, \\
\boldsymbol{n} \cdot\left(\boldsymbol{M} \nabla \mu^{\gamma}+\boldsymbol{h} \chi_{t}^{\gamma}\right)=0 & \text { on } S^{T}, \\
\mu^{\gamma}-\boldsymbol{g} \cdot \nabla \mu^{\gamma}=-\nabla \cdot \boldsymbol{\Gamma} \nabla \chi^{\gamma}+\psi^{\prime}\left(\chi^{\gamma}\right)+W_{, \chi}\left(\varepsilon\left(\boldsymbol{u}^{\gamma}\right), \chi^{\gamma}\right)+\beta \chi_{t}^{\gamma} & \text { in } \Omega^{T}, \\
\boldsymbol{n} \cdot \boldsymbol{\Gamma} \nabla \chi^{\gamma}=0 & \text { on } S^{T}, \\
\left.\mu^{\gamma}\right|_{t=0}=\mu_{0} & \text { in } \Omega,
\end{array}
$$

where $\mu_{0} \in L_{2}(\Omega)$. Suppose that

$$
\begin{aligned}
& \boldsymbol{u}^{m}(\boldsymbol{x}, t)=\sum_{i=1}^{m} e_{i}^{m}(t) \boldsymbol{v}_{i}(\boldsymbol{x}), \\
& \mu^{m}(\boldsymbol{x}, t)=\sum_{i=1}^{m} d_{i}^{m}(t) w_{i}(\boldsymbol{x}), \\
& \chi^{m}(\boldsymbol{x}, t)=\sum_{i=1}^{m} c_{i}^{m}(t) z_{i}(\boldsymbol{x})
\end{aligned}
$$

satisfy for a.e. $t \in[0, T]$ the identities with initial conditions

$$
\begin{aligned}
& \left\langle\boldsymbol{u}_{t t}^{\gamma, m}, \boldsymbol{v}_{j}\right\rangle_{\left(\boldsymbol{H}_{0}^{1}(\Omega)\right)^{\prime}, \boldsymbol{H}_{0}^{1}(\Omega)}+\left(\left(W_{, \varepsilon}\left(\varepsilon\left(\boldsymbol{u}^{\gamma, m}\right), \chi^{\gamma, m}\right), v_{j}\right)+\nu \boldsymbol{A}\left(\varepsilon\left(\boldsymbol{u}_{t}^{\gamma, m}\right), \boldsymbol{\varepsilon}\left(\boldsymbol{v}_{j}\right)\right)\right. \\
& =\left(\boldsymbol{b}, \boldsymbol{v}_{j}\right),\left.\quad \boldsymbol{u}^{\gamma, m}\right|_{t=0}=\boldsymbol{u}_{0}^{m},\left.\quad \boldsymbol{u}_{t}^{\gamma, m}\right|_{t=0}=\boldsymbol{u}_{1}^{m}, \\
& \gamma\left(\mu_{t}^{\gamma, m}, w_{j}\right)+\left(\chi_{t}^{\gamma, m}, w_{j}\right)+\left(\boldsymbol{M} \nabla \mu^{\gamma, m}+\boldsymbol{h} \chi_{t}^{\gamma, m}, \nabla w_{j}\right)=0, \\
& \left.\mu^{\gamma, m}\right|_{t=0}=\mu_{0}^{m} \text {, } \\
& \left(\mu^{\gamma, m}-\boldsymbol{g} \cdot \nabla \mu^{\gamma, m}, z_{j}\right)=\left(\boldsymbol{\Gamma} \nabla \chi^{\gamma, m}, \nabla z_{j}\right) \\
& +\left(\psi^{\prime}\left(\chi^{\gamma, m}\right)+W_{, \chi}\left(\varepsilon\left(\boldsymbol{u}^{\gamma, m}\right), \chi^{\gamma, m}\right)+\beta \chi_{t}^{\gamma, m}, z_{j}\right), \\
& \left.\chi^{\gamma, m}\right|_{t=0}=\chi_{0}^{m}
\end{aligned}
$$

for all $j=1, \ldots, m$, where

$$
\begin{aligned}
& \boldsymbol{u}_{0}^{m} \rightarrow \boldsymbol{u}_{0} \text { strongly in } \boldsymbol{H}_{0}^{1}(\Omega), \\
& \chi_{0}^{m} \rightarrow \chi_{0} \text { strongly in } H^{1}(\Omega), \\
& u_{1}^{m} \rightarrow u_{1}, \quad \mu_{0}^{m} \rightarrow \mu_{0} \quad \text { strongly in } L_{2}(\Omega) .
\end{aligned}
$$

The above regularization yields a well posed system for the coefficients $d_{i}^{m}(t), c_{i}^{m}(t), e_{i}^{m}(t)$, $i=1, \ldots, m$. Hence the system of ordinary differential equations (5.7) has a unique local solution.

Step 2. Estimates for Faedo-Galerkin approximation. Multiplying $(5.7)_{1}$ by $e_{j}^{m},(5.7)_{2}$ by $d_{j}^{m}$ and $(5.7)_{3}$ by $-c_{j}^{m}$, summing over $j$ from 1 to $m$ we obtain the equality 


$$
\begin{aligned}
& \frac{d}{d t}\left(\frac{\gamma}{2}\left\|\mu^{\gamma, m}\right\|_{L_{2}(\Omega)}^{2}+F\left(\boldsymbol{u}_{t}^{\gamma, m}, \boldsymbol{u}^{\gamma, m}, \chi^{\gamma, m}\right)\right) \\
& \quad+\nu \int_{\Omega} \boldsymbol{\varepsilon}\left(\boldsymbol{u}_{t}^{\gamma, m}\right) \cdot \boldsymbol{A} \boldsymbol{\varepsilon}\left(\boldsymbol{u}_{t}^{\gamma, m}\right) d x+\int_{\Omega}\left[\nabla \mu^{\gamma, m} \cdot \boldsymbol{M} \nabla \mu^{\gamma, m}+\chi_{t}^{\gamma, m}(\boldsymbol{g}+\boldsymbol{h}) \cdot \nabla \mu^{\gamma, m}\right. \\
& \left.\quad+\beta\left(\chi_{t}^{\gamma, m}\right)^{2}\right] d x=\int_{\Omega} \boldsymbol{b} \cdot \boldsymbol{u}_{t}^{\gamma, m} d x
\end{aligned}
$$

where $F$ is defined by (3.1). By the same arguments as presented formally in Section 3 we deduce the estimates

$$
\begin{aligned}
\gamma\left\|\mu^{\gamma, m}\right\|_{L_{\infty}\left(0, T ; L_{2}(\Omega)\right)}^{2} & +\frac{1}{2}\left\|\boldsymbol{u}_{t}^{\gamma, m}\right\|_{L_{\infty}\left(0, T ; \boldsymbol{L}_{2}(\Omega)\right)}^{2} \\
& +c_{1}\left\|\boldsymbol{u}^{\gamma, m}\right\|_{L_{\infty}\left(0, T ; \boldsymbol{H}_{0}^{1}(\Omega)\right)}^{2}+\frac{1}{2} \underline{c}_{\Gamma}\left\|\nabla \chi^{\gamma, m}\right\|_{L_{\infty}\left(0, T ; \boldsymbol{L}_{2}(\Omega)\right)}^{2} \\
& +\frac{1}{8}\left\|\chi^{\gamma, m}\right\|_{L_{\infty}\left(0, T ; L_{4}(\Omega)\right)}^{4}+c_{P}\left\|\nabla \mu^{\gamma, m}\right\|_{\boldsymbol{L}_{2}\left(\Omega^{T}\right)}^{2} \\
& +c_{P}\left\|\chi_{t}^{\gamma, m}\right\|_{L_{2}\left(\Omega^{T}\right)}^{2}+\nu c_{*}\left\|\varepsilon\left(\boldsymbol{u}_{t}^{\gamma, m}\right)\right\|_{\boldsymbol{L}_{2}\left(\Omega^{T}\right)}^{2} \leq \alpha_{1}
\end{aligned}
$$

and

$$
\begin{aligned}
& \left\|\mu^{\gamma, m}\right\|_{L_{2}\left(0, T ; H^{1}(\Omega)\right)}^{2} \leq c_{4}(1+T), \\
& \left\|\chi^{\gamma, m}\right\|_{L_{2}\left(0, T ; H^{2}(\Omega)\right)}^{2} \leq c_{5}(1+T), \\
& \left\|\boldsymbol{u}_{t t}^{\gamma, m}\right\|_{L_{2}\left(0, T ;\left(\boldsymbol{H}_{0}^{1}(\Omega)\right)^{\prime}\right)} \leq c_{6}(1+T),
\end{aligned}
$$

where $\alpha_{1}$ is defined in (2.17) and $c_{4}, c_{5}, c_{6}$ in (2.18).

Step 3. Passage to the limit $m \rightarrow \infty$. From the estimates (5.10), (5.11) it follows that there exist functions $\boldsymbol{u}^{\gamma}, \chi^{\gamma}, \mu^{\gamma}$ with

$$
\begin{aligned}
& \boldsymbol{u}^{\gamma} \in L_{\infty}\left(0, T ; \boldsymbol{H}_{0}^{1}(\Omega)\right), \quad \boldsymbol{u}_{t}^{\gamma} \in L_{\infty}\left(0, T ; \boldsymbol{L}_{2}(\Omega)\right), \quad \boldsymbol{u}_{t t}^{\gamma} \in L_{2}\left(0, T ;\left(\boldsymbol{H}_{0}^{1}(\Omega)\right)^{\prime}\right), \\
& \chi^{\gamma} \in L_{\infty}\left(0, T ; H^{1}(\Omega)\right) \cap L_{2}\left(0, T ; H^{2}(\Omega)\right), \\
& \mu^{\gamma} \in L_{2}\left(0, T ; H^{1}(\Omega)\right), \quad \gamma^{1 / 2} \mu^{\gamma} \in L_{\infty}\left(0, T ; L_{2}(\Omega)\right)
\end{aligned}
$$

and a subsequence of solutions $u^{\gamma, m}, \chi^{\gamma, m}, \mu^{\gamma, m}$ to (5.7) (which we still denote by the same indices) such that as $m \rightarrow \infty$ :

$$
\begin{array}{ll}
\boldsymbol{u}^{\gamma, m} \rightarrow \boldsymbol{u}^{\gamma} & \text { weakly-* in } L_{\infty}\left(0, T ; \boldsymbol{H}_{0}^{1}(\Omega)\right), \\
\boldsymbol{u}^{\gamma, m} \rightarrow \boldsymbol{u}_{t}^{\gamma} & \text { weakly-* in } L_{\infty}\left(0, T ; \boldsymbol{L}_{2}(\Omega)\right), \\
\boldsymbol{u}_{t t}^{\gamma, m} \rightarrow \boldsymbol{u}_{t t}^{\gamma} & \text { weakly in } L_{2}\left(0, T ;\left(\boldsymbol{H}_{0}^{1}(\Omega)\right)^{\prime}\right), \\
\chi^{\gamma, m} \rightarrow \chi^{\gamma} & \text { weakly-* in } L_{\infty}\left(0, T ; H^{1}(\Omega)\right) \text { and } \\
& \text { weakly in } L_{2}\left(0, T ; H^{2}(\Omega)\right), \\
\chi_{t}^{\gamma, m} \rightarrow \chi_{t}^{\gamma} & \text { weakly in } L_{2}\left(\Omega^{T}\right), \\
\mu^{\gamma, m} \rightarrow \mu^{\gamma} & \text { weakly in } L_{2}\left(0, T ; H^{1}(\Omega)\right), \\
\gamma^{1 / 2} \mu^{\gamma, m} \rightarrow \gamma^{1 / 2} \mu^{\gamma} & \text { weakly-* in } L_{\infty}\left(0, T ; L_{2}(\Omega)\right) .
\end{array}
$$


Then by the standard compactness results it follows that

$$
\begin{array}{ll}
\boldsymbol{u}^{\gamma, m} \rightarrow \boldsymbol{u}^{\gamma} & \text { strongly in } C\left([0, T] ; \boldsymbol{L}_{q}(\Omega)\right), \quad q<6, \text { and a.e in } \Omega^{T}, \\
\boldsymbol{u}_{t}^{\gamma, m} \rightarrow \boldsymbol{u}_{t}^{\gamma} & \text { strongly in } C\left([0, T] ;\left(\boldsymbol{H}_{0}^{1}(\Omega)\right)^{\prime}\right), \\
\chi^{\gamma, m} \rightarrow \chi^{\gamma} & \text { strongly in } L_{2}\left(0, T ; H^{1}(\Omega)\right) \cap C\left([0, T] ; L_{2}(\Omega)\right) \\
& \text { and a.e. in } \Omega^{T} .
\end{array}
$$

Hence,

$$
\begin{array}{ll}
\boldsymbol{u}^{\gamma, m}(0)=\boldsymbol{u}_{0}^{m} \rightarrow \boldsymbol{u}_{0} & \text { strongly in } \boldsymbol{L}_{q}(\Omega), \\
\boldsymbol{u}_{t}^{\gamma, m}(0)=\boldsymbol{u}_{1}^{m} \rightarrow \boldsymbol{u}_{1} & \text { strongly in }\left(H_{0}^{1}(\Omega)\right)^{\prime}, \\
\chi^{\gamma, m}(0)=\chi_{0}^{m} \rightarrow \chi_{0} & \text { strongly in } L_{2}(\Omega),
\end{array}
$$

which together with convergences (5.8) implies that

$$
\boldsymbol{u}^{\gamma}(0)=\boldsymbol{u}_{0}, \quad \boldsymbol{u}_{t}^{\gamma}(0)=\boldsymbol{u}_{1}, \quad \chi^{\gamma}(0)=\chi_{0} .
$$

We introduce the weak formulation corresponding to the Galerkin approximation (5.7),

$$
\begin{aligned}
& \int_{0}^{T}\left[\left\langle\boldsymbol{u}_{t t}^{\gamma,}, \boldsymbol{\eta}\right\rangle_{\left(\boldsymbol{H}_{0}^{1}(\Omega)\right)^{\prime}, \boldsymbol{H}_{0}^{1}(\Omega)}+\left(W_{, \varepsilon}\left(\varepsilon\left(\boldsymbol{u}^{\gamma, m}\right), \chi^{\gamma, m}\right), \boldsymbol{\eta}\right)\right. \\
& \left.\quad+\nu \boldsymbol{A}\left(\varepsilon\left(\boldsymbol{u}_{t}^{\gamma, m}\right), \boldsymbol{\varepsilon}(\boldsymbol{\eta})\right)\right] d t=\int_{0}^{T}(\boldsymbol{b}, \boldsymbol{\eta}) d t \\
& \int_{0}^{T}-\gamma\left(\mu^{\gamma, m}, \xi_{t}\right)+\left(\chi_{t}^{\gamma, m}, \xi\right)+\left(\boldsymbol{M} \nabla \mu^{\gamma, m}+h \chi_{t}^{\gamma, m}, \nabla \xi\right)=\gamma\left(\mu_{0}^{m}, \xi(0)\right), \\
& \int_{0}^{T}\left(\mu^{\gamma, m}-\boldsymbol{g} \cdot \nabla \mu^{\gamma, m}, \zeta\right) d t=\int_{0}^{T}\left[\left(\boldsymbol{\Gamma} \nabla \chi^{\gamma, m}, \nabla \zeta\right)\right. \\
& \left.\left.\quad+\psi^{\prime}\left(\chi^{\gamma, m}\right)+W_{, \chi}\left(\varepsilon\left(\boldsymbol{u}^{\gamma, m}\right), \chi^{\gamma, m}\right)+\beta \chi_{t}^{\gamma, m}, \zeta\right)\right] d t,
\end{aligned}
$$

where $\boldsymbol{\eta} \in L_{2}\left(0, T ; \boldsymbol{V}_{m}\right), \xi \in C^{1}\left([0, T], W_{m}\right), \zeta \in L_{2}\left(0, T ; Z_{m}\right)$ with $\xi(T)=0$.

In view of (5.12)-(5.14), following [BarPaw05], we can pass to the limit $m \rightarrow \infty$ in $(5.15)$.

Step 4. Passage to the limit $\gamma \rightarrow 0$. A priori estimates (5.10), (5.11) and the weak convergences (5.12) imply that the limit functions $\boldsymbol{u}^{\gamma}, \chi^{\gamma}, \mu^{\gamma}$ satisfy the estimates (5.10), (5.11) uniform in $\gamma$. Hence, we can deduce the convergences for $\gamma \rightarrow 0$ as in (5.12)-(5.14). Moreover, by virtue of the estimate

$$
\gamma^{1 / 2}\left\|\mu^{\gamma}\right\|_{L_{\infty}\left(0, T ; L_{2}(\Omega)\right)} \leq \alpha_{1}^{1 / 2}
$$

it follows that $\gamma \mu^{\gamma} \rightarrow 0$ strongly in $L_{\infty}\left(0, T ; L_{2}(\Omega)\right)$. Consequently, passing to the limit $\gamma \rightarrow 0$ in the integral identities for $u^{\gamma}, \chi^{\gamma}, \mu^{\gamma}$ analogous to (5.15) we obtain (2.16). This concludes the proof.

\section{Global existence}

Proof of Theorem 2.2. From (3.2) we deduce the continuity of $F(t)$, because

$$
\left|F\left(t^{\prime}\right)-F\left(t^{\prime \prime}\right)\right| \leq c\left|t^{\prime}-t^{\prime \prime}\right|^{1 / 2}\left(\int_{t^{\prime \prime}}^{t^{\prime}}\|\boldsymbol{b}(t)\|_{\boldsymbol{L}_{2}(\Omega)}^{2} d t\right)^{1 / 2},
$$


for $\left|t^{\prime}-t^{\prime \prime}\right|$ small. Moreover (3.6) implies that for any $k \in \mathbb{N} \cup\{0\}$

$$
\left\|\boldsymbol{u}_{t}(k T)\right\|_{\boldsymbol{L}_{2}(\Omega)}^{2}+\|\chi(k T)\|_{H^{1}(\Omega)}^{2}+\|\boldsymbol{u}(k T)\|_{\boldsymbol{H}^{1}(\Omega)}^{2} \leq 2 F(0)+\frac{3}{2}\|\boldsymbol{b}\|_{L_{1}\left(\mathbb{R}_{+} ; \boldsymbol{L}_{2}(\Omega)\right)}^{2}+c_{2} .
$$

Hence the local solution from Theorem 2.1 can be prolonged step by step on intervals $[k T,(k+1) T]$ up to $k=\infty$.

Before proving Theorem 2.3 we prepare a lemma. Let us simplify (4.4) to the form

$$
b_{4}(t)=\gamma_{1}\|\boldsymbol{b}(t)\|_{\boldsymbol{L}_{2}(\Omega)}^{2}+\gamma_{2}
$$

where $\gamma_{1}, \gamma_{2}$ are positive constants.

Lemma 6.1. Assume that

$$
\gamma_{3} \equiv \sup _{k \in \mathbb{N} \cup\{0\}}\|\boldsymbol{b}(t)\|_{L_{2}\left(k T,(k+1) T ; \boldsymbol{L}_{2}(\Omega)\right)}^{2}<\infty .
$$

Moreover, let $G(0)<\infty$, where $G$ is defined by (4.1). Then

$$
G(k T) \leq \frac{\gamma_{1} \gamma_{3}+\frac{1}{\delta_{*}} \gamma_{2}}{1-e^{-\delta_{*} T}}+e^{-k \delta_{*} T} G(0) \quad \text { for } \quad k \in \mathbb{N} \cup\{0\} .
$$

Proof. Multiplying (4.3) by $e^{\delta_{*} t}$ and integrating with respect to time from $i T$ to $(i+1) T$ we have

$$
G((i+1) T) \leq \gamma_{1} \gamma_{3}+\frac{1}{\delta_{*}} \gamma_{2}+e^{-\delta_{*} T} G(i T) .
$$

Integrating (6.3) with respect to $i$ from 0 to $k-1$ we obtain (6.2).

Proof of Theorem 2.3. From (4.3) we infer that $G(t)$ is continuous because

$$
\left|G\left(t^{\prime}\right)-G\left(t^{\prime \prime}\right)\right| \leq \gamma_{1} \int_{t^{\prime \prime}}^{t^{\prime}}\|\boldsymbol{b}(t)\|_{L_{2}(\Omega)}^{2} d t^{\prime}+\gamma_{2}\left(t^{\prime}-t^{\prime \prime}\right)
$$

for $\left|t^{\prime}-t^{\prime \prime}\right|$ small. Moreover, (6.2) implies that for any $k \in \mathbb{N}$,

$$
\|\boldsymbol{u}(k T)\|_{\boldsymbol{H}^{1}(\Omega)}^{2}+\left\|\boldsymbol{u}_{t}(k T)\right\|_{\boldsymbol{L}_{2}(\Omega)}^{2}+\|\chi(k T)\|_{H^{1}(\Omega)}^{2} \leq \frac{\gamma_{1} \gamma_{3}+\frac{1}{\delta_{*}} \gamma_{2}}{1-e^{-\delta_{*} T}}+e^{-k \delta_{*} T} G(0) .
$$

Hence the local solution from Theorem 2.1 can be prolonged step by step on intervals $[k T,(k+1) T]$ up to $k=\infty$.

7. Proof of Theorem 2.4. Let $\left(\boldsymbol{u}_{1}, \chi_{1}, \mu_{1}\right)$ and $\left(\boldsymbol{u}_{2}, \chi_{2}, \mu_{2}\right)$ be two solutions of (1.1)(1.3) (in simplified form (1.15)-(1.17)) corresponding to the same data. Subtracting the corresponding equations and denoting

$$
\boldsymbol{U}=\boldsymbol{u}_{1}-\boldsymbol{u}_{2}, \quad H=\chi_{1}-\chi_{2}, \quad Y=\mu_{1}-\mu_{2},
$$

yields the following system for $(\boldsymbol{U}, H, Y)$ :

$$
\begin{array}{ll}
\boldsymbol{U}_{t t}-\boldsymbol{Q} \boldsymbol{U}-\nu \boldsymbol{Q} \boldsymbol{U}_{t}=z^{\prime}\left(\chi_{1}\right) \boldsymbol{B} \nabla \chi_{1}-z^{\prime}\left(\chi_{2}\right) \boldsymbol{B} \nabla \chi_{2} & \text { in } \Omega^{T}, \\
\left.\boldsymbol{U}\right|_{t=0}=\mathbf{0},\left.\quad \boldsymbol{U}_{t}\right|_{t=0}=\mathbf{0} & \text { in } \Omega, \\
\boldsymbol{U}=\mathbf{0} & \text { on } S^{T},
\end{array}
$$




$$
\begin{array}{cl}
H_{t}-\nabla \cdot\left(\boldsymbol{M} \nabla Y+\boldsymbol{h} H_{t}\right)=0 & \text { in } \Omega^{T}, \\
\left.H\right|_{t=0}=0 & \text { in } \Omega, \\
\boldsymbol{n} \cdot\left(\boldsymbol{M} \nabla Y+\boldsymbol{h} H_{t}\right)=0 & \text { on } S^{T}, \\
Y-\boldsymbol{g} \cdot \nabla Y=-\nabla \cdot \boldsymbol{\Gamma} \nabla H+\psi^{\prime}\left(\chi_{1}\right)-\psi^{\prime}\left(\chi_{2}\right) & \\
+z^{\prime}\left(\chi_{1}\right)\left(\boldsymbol{B} \cdot \boldsymbol{\varepsilon}\left(\boldsymbol{u}_{1}\right)+D z\left(\chi_{1}\right)+E\right)-z^{\prime}\left(\chi_{2}\right)\left(\boldsymbol{B} \cdot \boldsymbol{\varepsilon}\left(\boldsymbol{u}_{2}\right)+D z\left(\chi_{2}\right)+E\right) & \text { in } \Omega^{T}, \\
+\beta H_{t} & \text { on } S^{T} . \\
\boldsymbol{n} \cdot \boldsymbol{\Gamma} \nabla H=0 &
\end{array}
$$

To get estimates we proceed similarly as in Section 3. Multiplying $(7.1)_{1}$ by $\boldsymbol{U}_{t}$, integrating over $\Omega$ and by parts, using boundary condition (7.1) 3 yields

$$
\begin{gathered}
\frac{1}{2} \frac{d}{d t}\left[\int_{\Omega}\left|\boldsymbol{U}_{t}\right|^{2} d x+\int_{\Omega} \varepsilon(\boldsymbol{U}) \cdot \boldsymbol{A} \varepsilon(\boldsymbol{U}) d x\right]+\nu \int_{\Omega} \varepsilon\left(\boldsymbol{U}_{t}\right) \cdot \boldsymbol{A} \varepsilon\left(\boldsymbol{U}_{t}\right) d x \\
=\int_{\Omega}\left(z^{\prime \prime}\left(\chi_{*}\right) H \boldsymbol{B} \nabla \chi_{1}+z^{\prime}\left(\chi_{2}\right) \boldsymbol{B} \nabla H\right) \cdot \boldsymbol{U}_{t} d x,
\end{gathered}
$$

where $\chi_{*} \in\left(\chi_{1}, \chi_{2}\right)$. Hence, recalling (2.2) and using assumptions on $z$,

$$
\begin{aligned}
& \frac{1}{2} \frac{d}{d t}\left(\left\|\boldsymbol{U}_{t}\right\|_{\boldsymbol{L}_{2}(\Omega)}^{2}+\int_{\Omega} \varepsilon(\boldsymbol{U}) \cdot \boldsymbol{A} \boldsymbol{\varepsilon}(\boldsymbol{U}) d x\right)+\nu c_{*}\left\|\varepsilon\left(\boldsymbol{U}_{t}\right)\right\|_{\boldsymbol{L}_{2}(\Omega)}^{2} \\
& \quad \leq c \int_{\Omega}\left|H\left\|\nabla \chi_{1}|| \boldsymbol{U}_{t}\left|d x+c \int_{\Omega}\right| \nabla H\right\| \boldsymbol{U}_{t}\right| d x .
\end{aligned}
$$

Next, we multiply $(7.2)_{1}$ by $Y$, integrate over $\Omega$ and by parts using $(7.2)_{3}$, to get

$$
\int_{\Omega} H_{t} Y d x+\int_{\Omega}\left(\nabla Y \cdot M \nabla Y+H_{t} \boldsymbol{h} \cdot \nabla Y\right) d x=0 .
$$

Further, we multiply $(7.3)_{1}$ by $-H_{t}$, integrate over $\Omega$ and by parts using $(7.3)_{2}$, to arrive at

$$
\begin{aligned}
\frac{1}{2} \frac{d}{d t} \int_{\Omega} \nabla H \cdot \boldsymbol{\Gamma} \nabla & H d x+\beta \int_{\Omega} H_{t}^{2} d x-\int_{\Omega} Y H_{t} d x+\int_{\Omega} H_{t} \boldsymbol{g} \cdot \nabla Y d x \\
= & -\int_{\Omega}\left(\psi^{\prime}\left(\chi_{1}\right)-\psi^{\prime}\left(\chi_{2}\right)\right) H_{t} d x-\int_{\Omega}\left[z ^ { \prime } ( \chi _ { 1 } ) \left(\boldsymbol{B} \cdot \boldsymbol{\varepsilon}\left(\boldsymbol{u}_{1}\right)\right.\right. \\
& \left.\left.+D z\left(\chi_{1}\right)+E\right)-z^{\prime}\left(\chi_{2}\right)\left(\boldsymbol{B} \cdot \boldsymbol{\varepsilon}\left(\boldsymbol{u}_{2}\right)+D z\left(\chi_{2}\right)+E\right)\right] H_{t} d x .
\end{aligned}
$$

Now, let us sum up (7.5) and (7.6). Taking advantage of structure condition (2.12), using that

$$
\psi^{\prime}\left(\chi_{1}\right)-\psi^{\prime}\left(\chi_{2}\right)=H\left(\chi_{1}^{2}+\chi_{1} \chi_{2}+\chi_{2}^{2}-1\right),
$$

and recalling assumptions on $z$, we deduce the inequality

$$
\begin{aligned}
& \frac{1}{2} \frac{d}{d t} \int_{\Omega} \nabla H \cdot \boldsymbol{\Gamma} \nabla H d x+c_{P}\left(\|\nabla Y\|_{\boldsymbol{L}_{2}(\Omega)}^{2}+\left\|H_{t}\right\|_{L_{2}(\Omega)}^{2}\right) \\
& \quad \leq c \int_{\Omega}|H|\left(\chi_{1}^{2}+\chi_{2}^{2}+1\right)\left|H_{t}\right| d x+c \int_{\Omega}|H|\left|\varepsilon\left(\boldsymbol{u}_{1}\right)\left\|H_{t}\left|d x+c \int_{\Omega}\right| \varepsilon(\boldsymbol{U})\right\| H_{t}\right| d x .
\end{aligned}
$$

Next, we estimate the mean value of $Y$. Since, by (7.2),

$$
\frac{d}{d t} \int_{\Omega} H d x=0 \quad \text { and } \quad \int_{\Omega} H d x=0 \text { for } t \in[0, T] \text {, }
$$


we deduce from (7.3) that

$$
\begin{aligned}
& \int_{\Omega} Y d x=\int_{\Omega} \boldsymbol{g} \cdot \nabla Y d x+\int_{\Omega}\left(\psi^{\prime}\left(\chi_{1}\right)-\psi^{\prime}\left(\chi_{2}\right)\right) d x \\
& \quad+\int_{\Omega}\left[z^{\prime}\left(\chi_{1}\right)\left(\boldsymbol{B} \cdot \boldsymbol{\varepsilon}\left(\boldsymbol{u}_{1}\right)+D z\left(\chi_{1}\right)+E\right)-z^{\prime}\left(\chi_{2}\right)\left(\boldsymbol{B} \cdot \boldsymbol{\varepsilon}\left(\boldsymbol{u}_{2}\right)+D z\left(\chi_{2}\right)+E\right)\right] d x .
\end{aligned}
$$

Hence,

$$
\begin{aligned}
\left|\int_{\Omega} Y d x\right| \leq & c \int_{\Omega}|\nabla Y| d x+c \int_{\Omega}|H|\left(\chi_{1}^{2}+\chi_{2}^{2}+1\right) d x \\
& +c \int_{\Omega}|H|\left|\varepsilon\left(\boldsymbol{u}_{1}\right)\right| d x+c \int_{\Omega}|\boldsymbol{\varepsilon}(\boldsymbol{U})| d x .
\end{aligned}
$$

Consequently, by the Cauchy-Schwarz inequality,

$$
\begin{aligned}
\left|\int_{\Omega} Y d x\right| \leq & c\|\nabla Y\|_{L_{2}(\Omega)}+c\left(\left\|\chi_{1}\right\|_{L_{4}}^{2}+\left\|\chi_{2}\right\|_{L_{4}(\Omega)}^{2}+1\right)\|H\|_{L_{2}(\Omega)} \\
& +c\left\|\varepsilon\left(\boldsymbol{u}_{1}\right)\right\|_{L_{2}(\Omega)}\|H\|_{L_{2}(\Omega)}+c\|\varepsilon(\boldsymbol{U})\|_{L_{2}(\Omega)} .
\end{aligned}
$$

From (7.10), owing to the Poincaré inequality (see (1.19)),

$$
\int_{\Omega} Y^{2} d x \leq c \int_{\Omega}|\nabla Y|^{2} d x+c\left(\int_{\Omega} Y d x\right)^{2},
$$

we deduce the estimate

$$
\begin{aligned}
\|Y\|_{H^{1}(\Omega)}^{2} & \leq c\|\nabla Y\|_{\boldsymbol{L}_{2}(\Omega)}^{2}+c\left(\left\|\chi_{1}\right\|_{L_{4}(\Omega)}^{4}+\left\|\chi_{2}\right\|_{L_{4}(\Omega)}^{4}+1\right)\|H\|_{L_{2}(\Omega)}^{2} \\
& +c\left\|\varepsilon\left(\boldsymbol{u}_{1}\right)\right\|_{\boldsymbol{L}_{2}(\Omega)}^{2}\|H\|_{L_{2}(\Omega)}^{2}+c\|\varepsilon(\boldsymbol{U})\|_{\boldsymbol{L}_{2}(\Omega)}^{2} .
\end{aligned}
$$

Finally, similarly as in Lemma 3.4, multiplying $(7.3)_{1}$ by $\Delta_{\boldsymbol{\Gamma}} H=\nabla \cdot \boldsymbol{\Gamma} \nabla H$, integrating over $\Omega$ and by parts using $(7.3)_{2}$, gives

$$
\begin{aligned}
\frac{\beta}{2} \frac{d}{d t} \int_{\Omega} \nabla H \cdot \boldsymbol{\Gamma} & \nabla H d x+\left\|\Delta_{\boldsymbol{\Gamma}} H\right\|_{L_{2}(\Omega)}^{2} \\
\leq & c \int_{\Omega}(|Y|+|\nabla Y|)\left|\Delta_{\boldsymbol{\Gamma}} H\right| d x+c \int_{\Omega}\left(\chi_{1}^{2}+\chi_{2}^{2}+1\right)\left|H \| \Delta_{\boldsymbol{\Gamma}} H\right| d x \\
& \quad+c \int_{\Omega}\left|\varepsilon\left(\boldsymbol{u}_{1}\right)\right|\left|H \| \Delta_{\boldsymbol{\Gamma}} H\right| d x+c \int_{\Omega}|\varepsilon(\boldsymbol{U})|\left|\Delta_{\boldsymbol{\Gamma}} H\right| d x .
\end{aligned}
$$

We shall show below that estimates (7.4), (7.7), (7.11) and (7.12) imply by Gronwall's lemma that $(\boldsymbol{U}, H, Y)=(\mathbf{0}, 0,0)$.

From (7.4), by the Hölder and Young inequalities, we deduce that

$$
\begin{aligned}
\frac{1}{2} \frac{d}{d t}\left(\left\|\boldsymbol{U}_{t}\right\|_{\boldsymbol{L}_{2}(\Omega)}^{2}+\int_{\Omega} \boldsymbol{\varepsilon}(\boldsymbol{U})\right. & \cdot \boldsymbol{A} \boldsymbol{\varepsilon}(\boldsymbol{U}) d x)+\nu c_{*}\left\|\boldsymbol{\varepsilon}\left(\boldsymbol{U}_{t}\right)\right\|_{\boldsymbol{L}_{2}(\Omega)}^{2} \\
\leq & \frac{\delta_{1}}{2}\left\|\boldsymbol{U}_{t}\right\|_{\boldsymbol{L}_{6}(\Omega)}^{2}+\frac{c}{2 \delta_{1}}\left\|\nabla \chi_{1}\right\|_{\boldsymbol{L}_{3 / 2}(\Omega)}^{2}\|H\|_{L_{6}(\Omega)}^{2} \\
& +\frac{\delta_{2}}{2}\left\|\boldsymbol{U}_{t}\right\|_{\boldsymbol{L}_{6}(\Omega)}^{2}+\frac{c}{2 \delta_{2}}\|\nabla H\|_{\boldsymbol{L}_{2}(\Omega)}^{2}, \quad \delta_{1}, \delta_{2}>0 .
\end{aligned}
$$

Hence, in view of Korn's inequality (1.18) and Sobolev imbedding (1.21), constants $\delta_{1}, \delta_{2}$ can be chosen so that the terms with $\boldsymbol{U}_{t}$ become absorbed by the left-hand side of (7.13). 
Thus

$$
\begin{aligned}
& \frac{1}{2} \frac{d}{d t}\left(\left\|\boldsymbol{U}_{t}\right\|_{\boldsymbol{L}_{2}(\Omega)}^{2}+\int_{\Omega} \varepsilon(\boldsymbol{U}) \cdot \boldsymbol{A} \varepsilon(\boldsymbol{U}) d x\right)+\frac{\nu c_{*}}{2}\left\|\varepsilon\left(\boldsymbol{U}_{t}\right)\right\|_{\boldsymbol{L}_{2}(\Omega)}^{2} \\
& \leq c\left\|\nabla \chi_{1}\right\|_{\boldsymbol{L}_{3 / 2}(\Omega)}^{2}\|H\|_{L_{6}(\Omega)}^{2}+c\|\nabla H\|_{\boldsymbol{L}_{2}(\Omega)}^{2} \leq c\left(\left\|\nabla \chi_{1}\right\|_{\boldsymbol{L}_{3 / 2}(\Omega)}^{2}+1\right)\|\nabla H\|_{\boldsymbol{L}_{2}(\Omega)}^{2},
\end{aligned}
$$

where in the last inequality we used the fact that, by the Sobolev imbedding and the Poincaré inequality, owing to (7.8),

$$
\|H\|_{L_{6}(\Omega)} \leq c\|H\|_{H^{1}(\Omega)} \leq c\|\nabla H\|_{L_{2}(\Omega)} .
$$

Next, let us turn to (7.7). Applying the Young inequality leads to

$$
\begin{aligned}
& \frac{1}{2} \frac{d}{d t} \int_{\Omega} \nabla H \cdot \boldsymbol{\Gamma} \nabla H d x+c_{P}\|\nabla Y\|_{\boldsymbol{L}_{2}(\Omega)}^{2}+\frac{c_{P}}{2}\left\|H_{t}\right\|_{L_{2}(\Omega)}^{2} \\
& \leq c \int_{\Omega} H^{2}\left(\chi_{1}^{4}+\chi_{2}^{4}+1\right) d x+c \int_{\Omega} H^{2}\left|\varepsilon\left(\boldsymbol{u}_{1}\right)\right|^{2} d x+c \int_{\Omega}|\varepsilon(\boldsymbol{U})|^{2} d x \equiv I_{1}+I_{2}+I_{3} .
\end{aligned}
$$

By the Hölder inequality,

$$
I_{1} \leq c\|H\|_{L_{6}(\Omega)}^{2}\left(\left\|\chi_{1}\right\|_{L_{6}(\Omega)}^{4}+\left\|\chi_{2}\right\|_{L_{6}(\Omega)}^{4}+1\right) .
$$

The term $I_{2}$ is bounded with the help of the interpolation inequality (see [BIN96]):

$$
\begin{aligned}
I_{2} & \leq c\|H\|_{L_{\infty}(\Omega)}^{2}\left\|\boldsymbol{\varepsilon}\left(\boldsymbol{u}_{1}\right)\right\|_{\boldsymbol{L}_{2}(\Omega)}^{2} \\
& \leq\left(\delta_{3}^{1 / 4}\|H\|_{H^{2}(\Omega)}^{2}+c \delta_{3}^{-3 / 4}\|H\|_{L_{2}(\Omega)}^{2}\right)\left\|\varepsilon\left(\boldsymbol{u}_{1}\right)\right\|_{\boldsymbol{L}_{2}(\Omega)}^{2},
\end{aligned}
$$

where $\delta_{3}>0$. We choose $\delta_{3}$ so that

$$
\delta_{3}^{1 / 4}\left\|\varepsilon\left(\boldsymbol{u}_{1}\right)\right\|_{\boldsymbol{L}_{2}(\Omega)}^{2}=\delta_{*}
$$

with $\delta_{*}>0$ to be selected later on. With such a choice of $\delta_{3}$,

$$
I_{2} \leq \delta_{*}\|H\|_{H^{2}(\Omega)}^{2}+c \delta_{*}^{-3}\left\|\varepsilon\left(\boldsymbol{u}_{1}\right)\right\|_{\boldsymbol{L}_{2}(\Omega)}^{8}\|H\|_{L_{2}(\Omega)}^{2} .
$$

The term $I_{3}$ is bounded by

$$
I_{3} \leq c\|\varepsilon(\boldsymbol{U})\|_{\boldsymbol{L}_{2}(\Omega)}^{2} .
$$

Combining estimates (7.17), (7.19), (7.20) in (7.16) and using (7.15) gives

$$
\begin{aligned}
\frac{1}{2} \frac{d}{d t} \int_{\Omega} \nabla H & \cdot \Gamma \nabla H d x+c_{P}\|\nabla Y\|_{L_{2}(\Omega)}^{2}+\frac{c_{P}}{2}\left\|H_{t}\right\|_{L_{2}(\Omega)}^{2} \\
\leq & c\left(\left\|\chi_{1}\right\|_{L_{6}(\Omega)}^{4}+\left\|\chi_{2}\right\|_{L_{6}(\Omega)}^{4}+1\right)\|\nabla H\|_{\boldsymbol{L}_{2}(\Omega)}^{2} \\
& +\delta_{*}\|H\|_{H^{2}(\Omega)}^{2}+c \delta_{*}^{-3}\left\|\varepsilon\left(\boldsymbol{u}_{1}\right)\right\|_{L_{2}(\Omega)}^{8}\|\nabla H\|_{L_{2}(\Omega)}^{2}+c \| \varepsilon\left(\boldsymbol{U} \|_{\boldsymbol{L}_{2}(\Omega)}^{2} .\right.
\end{aligned}
$$

Finally, let us turn to (7.12). Applying the Young inequality leads to

$$
\frac{\beta}{2} \frac{d}{d t} \int_{\Omega} \nabla H \cdot \Gamma \nabla H d x+\frac{1}{2}\left\|\Delta_{\Gamma} H\right\|_{L_{2}(\Omega)}^{2} \leq c\|Y\|_{H^{1}(\Omega)}^{2}+I_{1}+I_{2}+I_{3}
$$

with $I_{1}, I_{2}, I_{3}$ defined in (7.16).

The first term on the right-hand side of (7.22) is estimated by (7.11), $I_{1}$-term by (7.17), $I_{3}$-term by $(7.20)$, and $I_{2}$-term, similarly as in (7.18), by

$$
I_{2} \leq\left(\delta_{4}^{1 / 4}\|H\|_{H^{2}(\Omega)}^{2}+c \delta_{4}^{-3 / 4}\|H\|_{L_{2}(\Omega)}^{2}\right)\left\|\varepsilon\left(\boldsymbol{u}_{1}\right)\right\|_{\boldsymbol{L}_{2}(\Omega)}^{2}
$$

with a constant $\delta_{4}>0$ to be chosen in a moment. 
Combining the aforementioned estimates in (7.22) and using (7.15) we arrive at

$$
\begin{aligned}
& \frac{\beta}{2} \frac{d}{d t} \int_{\Omega} \nabla H \cdot \boldsymbol{\Gamma} \nabla H d x+\frac{1}{2}\left\|\Delta_{\boldsymbol{\Gamma}} H\right\|_{L_{2}(\Omega)}^{2} \\
& \leq c\|\nabla Y\|_{\boldsymbol{L}_{2}(\Omega)}^{2}+c\left(\left\|\chi_{1}\right\|_{L_{4}(\Omega)}^{4}+\left\|\chi_{2}\right\|_{L_{4}(\Omega)}^{4}+1\right)\|\nabla H\|_{\boldsymbol{L}_{2}(\Omega)}^{2} \\
& \quad+c\left\|\varepsilon\left(\boldsymbol{u}_{1}\right)\right\|_{\boldsymbol{L}_{2}(\Omega)}^{2}\|\nabla H\|_{\boldsymbol{L}_{2}(\Omega)}^{2}+c\|\boldsymbol{\varepsilon}(\boldsymbol{U})\|_{\boldsymbol{L}_{2}(\Omega)}^{2} \\
& \quad+\delta_{4}^{1 / 4}\left\|\boldsymbol{\varepsilon}\left(\boldsymbol{u}_{1}\right)\right\|_{\boldsymbol{L}_{2}(\Omega)}^{2}\|H\|_{H^{2}(\Omega)}^{2}+c \delta_{4}^{-3 / 4}\left\|\boldsymbol{\varepsilon}\left(\boldsymbol{u}_{1}\right)\right\|_{\boldsymbol{L}_{2}(\Omega)}^{2}\|\nabla H\|_{\boldsymbol{L}_{2}(\Omega)}^{2} .
\end{aligned}
$$

Let us note that owing to the ellipticity property of $\Delta_{\boldsymbol{\Gamma}}$ and the fact that $\int_{\Omega} H d x=0$, the following inequality holds true (see (3.27)):

$$
\|H\|_{H^{2}(\Omega)}^{2} \leq c_{1}\left\|\Delta_{\Gamma} H\right\|_{L_{2}(\Omega)}^{2}
$$

with a constant $c_{1}>0$. Thus, choosing $\delta_{4}$ so that

$$
\delta_{4}^{1 / 4} c_{1}\left\|\varepsilon\left(\boldsymbol{u}_{1}\right)\right\|_{\boldsymbol{L}_{2}(\Omega)}^{2}=\frac{1}{4},
$$

and consequently

$$
\delta_{4}^{-3 / 4}\left\|\varepsilon\left(\boldsymbol{u}_{1}\right)\right\|_{\boldsymbol{L}_{2}(\Omega)}^{2}=\left(4 c_{1}\right)^{3}\left\|\varepsilon\left(\boldsymbol{u}_{1}\right)\right\|_{\boldsymbol{L}_{2}(\Omega)}^{8},
$$

inequality (7.23) simplifies to

$$
\frac{\beta}{2} \frac{d}{d t} \int_{\Omega} \nabla H \cdot \Gamma \nabla H d x+\frac{1}{4}\left\|\Delta_{\Gamma} H\right\|_{L_{2}(\Omega)}^{2}
$$

$\leq c_{2}\|\nabla Y\|_{\boldsymbol{L}_{2}(\Omega)}^{2}+c\left(\left\|\chi_{1}\right\|_{L_{4}(\Omega)}^{4}+\left\|\chi_{2}\right\|_{L_{4}(\Omega)}^{4}+\left\|\varepsilon\left(\boldsymbol{u}_{1}\right)\right\|_{\boldsymbol{L}_{2}(\Omega)}^{8}+1\right)\|\nabla H\|_{\boldsymbol{L}_{2}(\Omega)}^{2}+c\|\varepsilon(\boldsymbol{U})\|_{\boldsymbol{L}_{2}(\Omega)}^{2}$, where we distinguished constant $c_{2}$ in front of the first term on the right-hand side of (7.25). Now, multiplying (7.25) by $\frac{c_{P}}{2 c_{2}}$ and adding to (7.21), we get

$$
\begin{aligned}
\left(\frac{\beta c_{P}}{4 c_{2}}+\frac{1}{2}\right) \frac{d}{d t} & \int_{\Omega} \nabla H \cdot \boldsymbol{\Gamma} \nabla H d x+\frac{c_{P}}{8 c_{2}}\left\|\Delta_{\boldsymbol{\Gamma}} H\right\|_{L_{2}(\Omega)}^{2} \\
& +\frac{c_{P}}{2}\left(\|\nabla Y\|_{\boldsymbol{L}_{2}(\Omega)}^{2}+\left\|H_{t}\right\|_{L_{2}(\Omega)}^{2}\right) \\
\leq & c\left(\left\|\chi_{1}\right\|_{L_{6}(\Omega)}^{4}+\left\|\chi_{2}\right\|_{L_{6}(\Omega)}^{4}+\left\|\varepsilon\left(\boldsymbol{u}_{1}\right)\right\|_{\boldsymbol{L}_{2}(\Omega)}^{8}+1\right)\|\nabla H\|_{\boldsymbol{L}_{2}(\Omega)}^{2} \\
& +c\|\varepsilon(\boldsymbol{U})\|_{\boldsymbol{L}_{2}(\Omega)}^{2}+\delta_{*}\|H\|_{H^{2}(\Omega)}^{2}+c \delta_{*}^{-3}\left\|\varepsilon\left(\boldsymbol{u}_{1}\right)\right\|_{\boldsymbol{L}_{2}(\Omega)}^{8}\|\nabla H\|_{\boldsymbol{L}_{2}(\Omega)}^{2} .
\end{aligned}
$$

In view of (7.23), choosing constant $\delta_{*}=\frac{c_{P}}{16 c_{1} c_{2}}$, the last but one term on the right-hand side of (7.26) is absorbed by its left-hand side. Adding the resulting inequality to (7.14), we finally arrive at

$$
\begin{aligned}
\frac{1}{2} \frac{d}{d t}\left(\left\|\boldsymbol{U}_{t}\right\|_{\boldsymbol{L}_{2}(\Omega)}^{2}+\right. & \left.\int_{\Omega} \boldsymbol{\varepsilon}(\boldsymbol{U}) \cdot \boldsymbol{A} \boldsymbol{\varepsilon}(\boldsymbol{U}) d x\right) \\
& +\left(\frac{\beta c_{P}}{4 c_{2}}+\frac{1}{2}\right) \frac{d}{d t} \int_{\Omega} \nabla H \cdot \boldsymbol{\Gamma} \nabla H d x+\frac{\nu c_{*}}{2}\left\|\varepsilon\left(\boldsymbol{U}_{t}\right)\right\|_{\boldsymbol{L}_{2}(\Omega)}^{2} \\
& +\frac{c_{8}}{16 c_{2}}\left\|\Delta_{\boldsymbol{\Gamma}} H\right\|_{L_{2}(\Omega)}^{2}+\frac{c_{P}}{2}\left(\|\nabla Y\|_{\boldsymbol{L}_{2}(\Omega)}^{2}+\left\|H_{t}\right\|_{L_{2}(\Omega)}^{2}\right) \\
\leq & c\left(\left\|\chi_{1}\right\|_{L_{6}(\Omega)}^{4}+\left\|\chi_{2}\right\|_{L_{6}(\Omega)}^{4}+\left\|\varepsilon\left(\boldsymbol{u}_{1}\right)\right\|_{\boldsymbol{L}_{2}(\Omega)}^{8}\right. \\
& \left.+\left\|\nabla \chi_{1}\right\|_{\boldsymbol{L}_{3 / 2}(\Omega)}^{2}+1\right)\|\nabla H\|_{\boldsymbol{L}_{2}(\Omega)}^{2}+c\|\varepsilon(\boldsymbol{U})\|_{\boldsymbol{L}_{2}(\Omega)}^{2} .
\end{aligned}
$$


Denoting

$$
\begin{gathered}
D(t)=\frac{1}{2}\left(\left\|\boldsymbol{U}_{t}\right\|_{\boldsymbol{L}_{2}(\Omega)}^{2}+\int_{\Omega} \boldsymbol{\varepsilon}(\boldsymbol{U}) \cdot \boldsymbol{A} \boldsymbol{\varepsilon}(\boldsymbol{U}) d x\right)+\left(\frac{\beta c_{P}}{4 c_{2}}+\frac{1}{2}\right) \int_{\Omega} \nabla H \cdot \boldsymbol{\Gamma} \nabla H d x, \\
p(t)=c\left(\left\|\chi_{1}\right\|_{L_{6}(\Omega)}^{4}+\left\|\chi_{2}\right\|_{L_{6}(\Omega)}^{4}+\left\|\boldsymbol{\varepsilon}\left(\boldsymbol{u}_{1}\right)\right\|_{\boldsymbol{L}_{2}(\Omega)}^{8}+\left\|\nabla \chi_{1}\right\|_{\boldsymbol{L}_{3 / 2}(\Omega)}^{2}+1\right),
\end{gathered}
$$

and recalling $(2.2),(2.6)$, it follows from (7.27) that

$$
\begin{aligned}
\frac{d}{d t} D(t)+\frac{\nu c_{*}}{2}\left\|\varepsilon\left(\boldsymbol{U}_{t}\right)\right\|_{\boldsymbol{L}_{2}(\Omega)}^{2}+\frac{c_{8}}{16 c_{2}} & \left\|\Delta_{\boldsymbol{\Gamma}} H\right\|_{L_{2}(\Omega)}^{2} \\
& +\frac{c_{P}}{2}\left(\|\nabla Y\|_{L_{2}(\Omega)}^{2}+\left\|H_{t}\right\|_{L_{2}(\Omega)}^{2}\right) \leq p(t) D(t) .
\end{aligned}
$$

Hence, by the Gronwall lemma,

$$
D(t) \leq D(0) \exp \int_{0}^{t} p\left(t^{\prime}\right) d t^{\prime} .
$$

Since $D(0)=0$, and due to energy estimates $(2.17)$

$$
\begin{aligned}
\int_{0}^{t} p\left(t^{\prime}\right) d t^{\prime} \leq & c\left(\left\|\chi_{1}\right\|_{L_{\infty}\left(0, T ; H^{1}(\Omega)\right)}+\left\|\chi_{2}\right\|_{L_{\infty}\left(0, T ; H^{1}(\Omega)\right)}\right. \\
& \left.+\left\|\boldsymbol{u}_{1}\right\|_{L_{\infty}\left(0, T ; H^{1}(\Omega)\right)}^{8}+1\right) T \leq c T<\infty,
\end{aligned}
$$

we conclude that $D(t)=0$ for $t \in[0, T]$. Moreover, from (7.28) it follows that

$$
\left\|\varepsilon\left(\boldsymbol{U}_{t}\right)\right\|_{\boldsymbol{L}_{2}(\Omega)}=\left\|\Delta_{\boldsymbol{\Gamma}} H\right\|_{\boldsymbol{L}_{2}(\Omega)}=\left\|H_{t}\right\|_{L_{2}(\Omega)}=\|\nabla Y\|_{\boldsymbol{L}_{2}(\Omega)}=0 .
$$

Hence, $\boldsymbol{U}=\mathbf{0}$ and $H=0$ in $\Omega^{T}$, and by $(7.11),\|Y\|_{H^{1}(\Omega)}=0$, so that $Y=0$ in $\Omega^{T}$. This proves the uniqueness of the solution $(\boldsymbol{u}, \chi, \mu)$ in the interval $[0, T]$.

\section{References}

[BarPaw05] E. Bartkowiak and I. Pawłow, The Cahn-Hilliard-Gurtin system coupled with elasticity, Control and Cybernetics 34 (2005), 1005-1043.

[BCDGSS02] E. Bonetti, P. Colli, W. Dreyer, G. Giliardi, G. Schimperna and J. Sprekels, On a model for phase separation in binary alloys driven by mechanical effects, Physica D 165 (2002), 48-65.

[BIN96] O. V. Besov, V. P. Il'in and S. M. Nikolski, Integral Representations of Functions and Imbedding Theorems, Nauka, Moscow, 1996 (in Russian).

[CarMirP00] M. Carrive, A. Miranville and A. Piétrus, The Cahn-Hilliard equation for deformable elastic continua, Adv. Math. Sci. Appl. 10 (2002), 539-569.

[CarMirPR99] M. Carrive, A. Miranville, A. Piétrus and J. Rakotoson, The Cahn-Hilliard equation for anisotropic deformable elastic continum, Appl. Math. Lett. 12 (1999), 23-28.

[Gar00] H. Garcke, On mathematical models for phase separation in elastically stressed solids, Habilitation Thesis, University of Bonn, 2000.

[Gar03] -, On Cahn-Hilliard system with elasticity, Proc. Roy. Soc. Edinburgh 133A (2003), 307-331.

[Gar05] - On a Cahn-Hilliard model for phase separation with elastic misfit, Ann. Inst. H. Poincaré Anal. Non Linéaire 22 (2005), 165-185. 
[Gur96] M. E. Gurtin, Generalized Ginzburg-Landau and Cahn-Hilliard equations based on a microforce balance, Physica D 92 (1996), 178-192.

[Mir00] A. Miranville, Some generalizations of Cahn-Hilliard equation, Asymptotic Analysis 22 (2000), 235-259.

[Mir01a] A. Miranville, Long-time behavior of some models of Cahn-Hilliard equations in deformable continua, Nonlinear Analysis: Real World Applications 2 (2001), 273-304.

[Mir01b] A. Miranville, Consistent models of Cahn-Hilliard-Gurtin equations with Neumann boundary conditions, Physica D 158 (2001), 233-257.

[Paw06] I. Pawłow, Thermodynamically consistent Cahn-Hilliard and Allen-Cahn models in elastic solids, Discrete and Continuous Dynamical Systems 15, (2006), 1169-1191.

[PawZaj06a] I. Pawłow and W. M. Zajączkowski, Classical solvability of 1-D Cahn-Hilliard equation coupled with elasticity, Mathematical Methods in the Applied Sciences 29 (2006), 853-876.

[PawZaj06b] I. Pawłow and W. M. Zajączkowski, Weak solutions to 3-D Cahn-Hilliard system in elastic solids, Topological Methods in Nonlinear Analysis (to appear).

[PawZaj07] I. Pawłow and W. M. Zajączkowski, Strong solvability of 3-D Cahn-Hilliard system in elastic solids, Mathematical Methods in the Applied Sciences (to appear). 